Library, N.W. Bldg

OCT 191964

NBS MONOGRAPH 78

\title{
A Complete Mode Sum for LF, VLF, ELF Terrestrial Radio Wave Fields
}

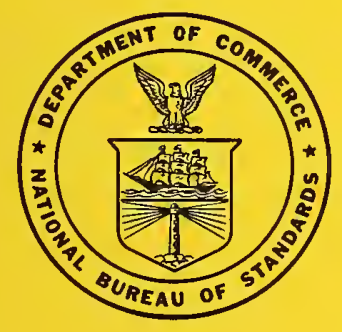

\section{U.S. DEPARTMENT OF COMMERCE}

NATIONAL BUREAU OF STANDARDS 


\section{THE NATIONAL BUREAU OF STANDARDS}

The National Bureau of Standards is a principal focal point in the Federal Government for assuring maximum application of the physical and engineering sciences to the advancement of technology in industry and commerce. Its responsibilities include development and maintenance of the national standards of measurement, and the provisions of means for making measurements consistent with those standards; determination of physical constants and properties of materials; development of methods for testing materials, mechanisms, and structures, and making such tests as may be necessary, particularly for government agencies; cooperation in the establishment of standard practices for incorporation in codes and specifications; advisory service to government agencies on scientific and technical problems; invention and development of devices to serve special needs of the Government; assistance to industry, business, and consumers in the development and acceptance of commercial standards and simplified trade practice recommendations; administration of programs in cooperation with United States business groups and standards organizations for the development of international standards of practice; and maintenance of a clearinghouse for the collection and dissemination of scientific, technical, and engineering information. The scope of the Bureau's activities is suggested in the following listing of its four Institutes and their organizational units.

Institute for Basic Standards. Electricity. Metrology. Heat. Radiation Physics. Mechanics. Applied Mathematics. Atomic Physics. Physical Chemistry. Laboratory Astrophysics." Radio Standards Laboratory: Radio Standards Physics; Radio Standards Engineering." * Office of Standard Reference Data.

Institute for Materials Research. Analytical Chemistry. Polymers. Metallurgy. Inorganic Materials. Reactor Radiations. Cryogenics.* * Office of Standard Reference Materials.

Central Radio Propagation Laboratory.** Ionosphere Research and Propagation. Troposphere and Space Telecommunications. Radio Systems. Upper Atmosphere and Space Physics.

Institute for Applied Technology. Textiles and Apparel Technology Center. Building Research. Industrial Equipment. Information Technology. Performance Test Development. Instrumentation. Transport Systems. Office of Technical Services. Office of Weights and Measures. Office of Engineering Standards. Office of Industrial Services.

\footnotetext{
* NBS Group, Joint Institute for Laboratory Astrophysics at the University of Colorado.

* Located at Boulder, Colorado.
} 
UNITED STATES DEPARTMENT OF COMMERCE - Luther H. Hodges, Secretary NATIONAL BUREAU OF STANDARDS - A. V. Astin, Director

\section{A Complete Mode Sum for LF, VLF, ELF Terrestrial Radio Wave Fields}

J. Ralph Johler and Leslie A. Berry

Central Radio Propagation Laboratory

National Bureau of Standards

Boulder, Colorado

National Bureau of Standards Monograph 78

Issued October 1, 1964 


\section{Contents}

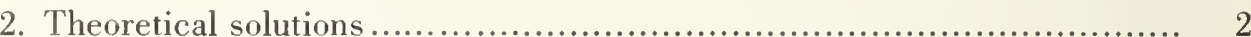

2.1. Isotropic model ionosphere.......................................... 4

2.2. Anisotropic solution with constant radial magnetic field ............... 5

2.3. Reflection coefficients............................................. 8

3. Computational methods ................................................ 12

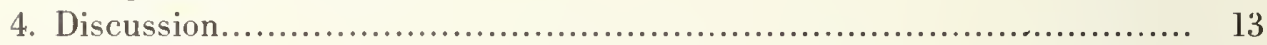

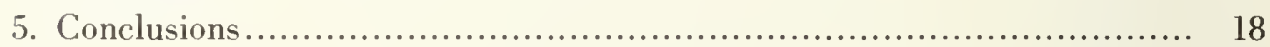

6. References ............................................................... 19

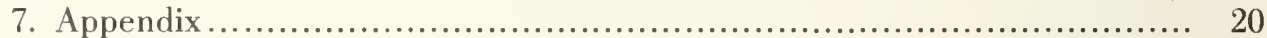




\title{
A Complete Mode Sum for LF, VLF, ELF Terrestrial Radio Wave Fields ${ }^{1}$
}

\author{
J. Ralph Johler and Leslie A. Berry \\ Contribution From the Central Radio Propagation Laboratory, \\ National Bureau of Standards, Boulder, Colo.
}

\begin{abstract}
Previous papers about VLF, ELF propagation in the earth-ionosphere waveguide have emphasized the characteristics of the individual modes. In this paper, emphasis is on the total field found by summing all modes which contribute appreciably. Solutions are given and calculations are made for a homogeneous isotropic ionosphere, a homogeneous ionosphere with a constant radial magnetic field imposed, and an ionosphere with a magnetic field of arbitrary dip angle.

In the isotropic case, one mode is sufficient to describe the field at ELF, but at the upper end of the VLF band, two or more modes may propagate $7500 \mathrm{~km}$. When a magnetic field is imposed on the ionosphere, three abnormal modes or fields are generated at the anisotropic boundary. These abnormal modes have little effect on the total field in the VLF band, but they change the ELF field appreciably. Indeed, in the absorption region between VLF and ELF, the abnormal modes may become dominant beyond $1000 \mathrm{~km}$.
\end{abstract}

\section{Introduction}

The object of this analysis is a more accurate description of the field of the LF, VLF, ELF radio wave in the space between the ionosphere and the terrestrial sphere. A number of investigators have devised theoretical methods to this end; indeed, considerable emphasis has been placed on the development of field-strength curves, both as a function of distance and frequency. Wait and Murphy [1957] published field-strength curves computed with the aid of the geometric-optical theory, applicable to short distances, and Wait [1957, 1962] and Wait and Carter [1960] developed curves with the aid of the residue methods, or "mode theory," which is a full-wave solution for an assumed model, but employed the Debye [1910] approximation for the spherical-wave function. This approximation was used previously by March [1912] and von Rybczyński [1913] for the groundwave problem and is, as pointed out by March [1912], not valid in areas corresponding to the most important terms in the residue series at frequencies greater than 8 or $10 \mathrm{kc} / \mathrm{s}$. It is interesting to note that von Rybczyński [1913] analyzed and corrected March's [1912] paper in the use of this approximation. Budden [196la] considered the full-wave solutions or "mode characteristics" assuming a flat model for the terrestrial sphere and ionosphere, with the implication that this procedure simplified the analysis. Alpert [1961] finds the mode sum for a flat waveguide and then multiplies by a geometric factor to correct for sphericity. Wait [1961] computed the attenuation and phase velocity of the least attenuated residue for certain specified models, including in the solution some of the effects of anisotropy, and Wait and Spies [1963] considered the amplitude of the "excitation factor" of the first two residues with a perfectly conducting earth and ionosphere and of the first residue for a finitely conducting ionosphere.

The possible multimode character of the guide has been noted by Wait [1962a, b]; however, since large scale computer techniques have not been available, little has been done to sum all modes. Indeed, at distances less than about $3000 \mathrm{~km}$, it is not sufficient to consider only a single such mode, if the frequency is greater than about $15 \mathrm{kc} / \mathrm{s}$, for a daytime-noon ionosphere model employed by Johler and Berry $[1962,1963]$ or at frequencies greater than about 4 or $5 \mathrm{kc} / \mathrm{s}$ for a corresponding nighttime model ionosphere. Johler and Berry [1962, 1963] computed LF, VLF, ELF fields by summing the classical series of zonal harmonics and by summing the residues for

${ }^{1}$ This paper was presented at the VLF Symposium, Boulder, Colo., on August 12, 1963. 
the contour integral of the Watson transformation (called "modes" by Wait [1962a, b] and by others). The present paper extends the previous work of Johler and Berry [1962] to take account of anisotropy. Curves are presented for the field which include all residues or modes of signifcance to the computations.

\section{Theoretical Solutions}

The electromagnetic propagation of radio waves in an anistropic medium is described by Maxwell's classical equations. For the continuous time-harmonic solution, with a frequency, $f=\omega / 2 \pi$,

$$
\begin{gathered}
\frac{\partial}{\partial t} \vec{E}=i \omega \vec{E}, \frac{\partial}{\partial t} \vec{H}=i \omega \vec{H} \\
\nabla \times \vec{E}+\mu_{0} i \omega \vec{H}=0 \\
\nabla \times \vec{H}-[\epsilon] \cdot \vec{E}=0,
\end{gathered}
$$

where $\vec{E}$ and $\vec{H}$ are the electric and magnetic intensities (rationalized mks units) and $\mu_{0}, \epsilon_{0}$ are the permeability and permittivity $\left(\epsilon_{0}=1 / c^{2} \mu_{0}\right.$ where $c$ is the speed of light) of space and [ $\epsilon$ ] is the dielectric tensor of the anisotropic medium. In the isotropic, nonconducting case,

$$
[\epsilon]=\epsilon i \omega=\epsilon_{0} i \omega \text { in free space. }
$$

The dielectric tensor in an anistropic medium, employing the Cartesian coordinate systems, $x, y, z$, figure 1, can be written [Johler and Harper, 1962; Johler, 1963a, 1963b]

$$
\begin{aligned}
& {\left[i \omega+\omega_{N}^{2}\left[\frac{\frac{1}{2}}{\nu+i\left(\omega+\omega_{H}\right)}+\frac{\frac{1}{2}}{\nu+i\left(\omega-\omega_{H}\right)}\right] \omega_{N}^{2}\left[\frac{\frac{i}{2} \sin I}{\nu+i\left(\omega+\omega_{H}\right)}+\frac{\frac{-i}{3} \sin I}{\nu+i\left(\omega-\omega_{H}\right)}\right] \quad . \quad \omega_{N}^{2}\left[\frac{\frac{i}{2} \cos I}{\nu+i\left(\omega+\omega_{H}\right)}+\frac{\frac{-i}{2} \cos I}{\nu+i\left(\omega-\omega_{H}\right)}\right]\right.} \\
& \omega_{N}^{2}\left[\frac{\frac{-i}{2} \sin I}{\nu+i\left(\omega+\omega_{H}\right)}+\frac{\frac{i}{3} \sin I}{\nu+i\left(\omega-\omega_{H}\right)}\right] \quad i \omega+\omega_{N}^{2}\left[\frac{\cos ^{2} I}{\nu+i \omega}+\frac{\frac{1}{2} \sin ^{2} I}{\nu+i\left(\omega+\omega_{H}\right)}+\frac{\frac{1}{2} \sin ^{2} I}{\nu+i\left(\omega-\omega_{H}\right)}\right] \quad \omega_{N}^{2}\left[\frac{-\sin I \cos I}{\nu+i \omega}+\frac{\frac{1}{2} \sin I \cos I}{\nu+i\left(\omega+\omega_{H}\right)}+\frac{\frac{1}{2} \sin I \cos I}{\nu+i\left(\omega-\omega_{H}\right)}\right](3 \\
& \omega_{\psi}^{2}\left[\frac{\frac{-i}{2} \cos I}{\nu+i\left(\omega+\omega_{H}\right)}+\frac{\frac{i}{2} \cos I}{\nu+i\left(\omega-\omega_{H}\right)}\right] \omega_{N}^{2}\left[\frac{-\sin I \cos I}{\nu+i \omega}+\frac{\frac{1}{2} \sin I \cos I}{\nu+i\left(\omega+\omega_{H}\right)}+\frac{\frac{1}{2} \sin I \cos I}{\nu+i\left(\omega-\omega_{H}\right)}\right] i \omega+\omega_{N}^{2}\left[\frac{\sin ^{2} I}{\nu+i \omega}+\frac{\frac{1}{2} \cos ^{2} I}{\nu+i\left(\omega+\omega_{H}\right)}+\frac{\frac{1}{2} \cos ^{2} I}{\nu+i\left(\omega-\omega_{H}\right)}\right]
\end{aligned}
$$

The motion of the electron is described by the Langevin equation,

$$
m i \omega \vec{V}+m g \vec{V}+e(\vec{V} \times \vec{B})+e \vec{E}=0
$$

where the complex, frequency-dependent parameter, $g$, is assumed to be a constant, $g \cong \nu$ [Johler, 1963a] where $\omega_{\mathrm{H}}=\mu_{0} e \mathscr{H} / m$, the gyrofrequency for electron charge, $e$, mass, $m$, and the magnetic field, $\mathscr{H}=|\overrightarrow{\mathscr{H}}|$ or the magnetic induction $\vec{B}=\mu_{0} \overrightarrow{\mathscr{H}} . \quad \omega_{\mathrm{N}}^{2}=N e^{2} / \epsilon_{0} m$, the angular plasma frequency squared, $I$ is the magnetic dip measured from the horizontal, $N$ is the electron density of the plasma and the magnetic field, $\overrightarrow{\mathscr{H}}$, is assumed to be oriented in the $y$ - $z$ plane.

If a plane wave,

$$
E=A \exp \left[i \omega t-i \frac{\omega}{c} \eta D\right]
$$

where

$$
\eta D=x \sin \phi_{i} \sin \phi_{a}+y \sin \phi_{i} \cos \phi_{a}+z \zeta
$$


FIGURE 1. Cartesian coordinate system and spherical geometry.

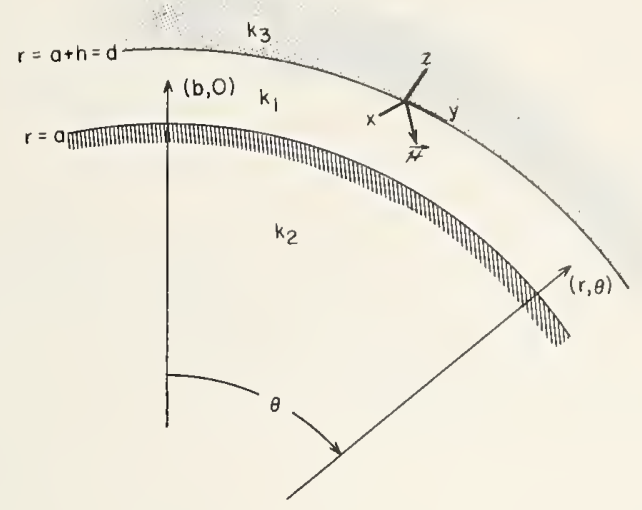

is assumed as a solution of Maxwell's equations in such a medium, where $\phi_{i}$ is the angle of incidence relative to the vertical $z$-direction, and $\phi_{a}$ is the magnetic azimuth measured clockwise from the $y$ - $z$ plane containing the magnetic vector, $\vec{H}$, with a dip angle $I$ from the horizontal, a quartic in the complex number, $\zeta$, is deduced [Johler and Walters, 1960; Booker, 1939]

$$
a_{4} \zeta^{4}+a_{3} \zeta^{3}+a_{2} \zeta^{2}+a_{1} \zeta^{1}+a_{0}=0 .
$$

The coefficients of this quartic are given in appendix 1 , and a complex index of refraction, $\eta$, of the medium is defined,

$$
\eta^{2}=\zeta^{2}+\sin ^{2} \phi_{i}
$$

Equation (6) is analogous to Booker's [1939] quartic in classical magneto-ionic theory, because Booker first attached physical significance to the four roots as: ordinary $(o)$ and extraordinary $(e)$, upgoing (i) and downgoing $(r)$ propagation components, $\eta=\eta_{o}^{i,},{ }_{e}$. The downgoing components can arise from boundaries (more than one) or electron density gradients in the $z$-direction. Thus, in the homogeneous medium, only upgoing ordinary and extraordinary propagation components exist.

Attempts to solve Maxwell's equations in an anisotropic medium in coordinate systems other than Cartesian, such as spherical, $r, \theta, \phi$, figure 1 , coordinate systems lead to intractable equations by conventional methods. However, for propagation theory in the space between the plasma and the terrestrial sphere, approximate solutions can be worked out and tested, which task is an objective of this analysis.

The spherical geometry of the model propagation media is shown in figure 1 , together with the transmitter, receiver, and boundaries. The origin of a spherical coordinate system $(r, \theta, \phi)$ is the center of a homogeneous terrestrial sphere with a radius, $a$, and a wave number,

$$
k_{2}=\frac{\omega}{c} \sqrt{\epsilon_{2}-i \frac{\sigma \mu_{0} c^{2}}{\omega}}
$$

where $\epsilon_{2}$ is the dielectric constant relative to a vacuum, or the permittivity, $\epsilon=\epsilon_{2} \epsilon_{0}$ and $\sigma$ is the conductivity (ohms $-1 /$ meter) of the terrestrial sphere. The space between the ionosphere and the terrestrial sphere is represented electrically by the wave number,

$$
k_{1}=\frac{\omega}{c} \eta_{1}
$$

where $\eta_{1}$ is the index of refraction $\left(\eta_{1} \sim 1\right.$ for vacuum, $\eta_{1} \sim 1.000338$ for air at the surface of the earth). 
A vertical electric Hertzian (electrical point source) dipole is located at $\theta=0, r=b(\mathrm{a}<b<d)$, and the field is to be calculated at any point $(r, \theta, \phi)$, although the specializations in this analysis confine the computations to the space $(a<r<d)$. Azimuthal symmetry will be assumed $\left(\frac{\partial}{\partial \phi}=0\right)$.

\section{1. Isotropic Model Ionosphere}

When the earth's magnetic field is neglected, the ionosphere can be characterized by the isotropic wave number

$$
k_{3}=\frac{\omega}{c} \sqrt{1-i \frac{\omega_{N}^{2}}{\omega(\nu+i \omega)}}
$$

The solution for this case is well known [Watson, 1918].

The vertical electric field, in particular, can be written, using the $\mathbb{W}$ atson transformation, ${ }^{2}$

$$
E_{r}=\left[\frac{\mu_{0} c}{4 \pi} I_{0} l\right]\left[\frac{-\pi}{k_{1}^{2} r^{2} b^{2}}\right] \sum_{s=1}^{\infty} \frac{\left(v_{s}^{2}-\frac{1}{4}\right)\left(v_{s}\right) P_{i s-1 / 2}(-\cos \theta)}{\cos v_{s} \pi} \frac{A v_{s-1 / 2}}{\left\{\frac{\partial}{\partial v} D_{v-1 / 2}\right\}_{v=v_{s}}}
$$

where $P_{n}(\cos \theta)$ is the Legendre function,

$$
\begin{gathered}
\zeta_{v}^{(i)}(z)=\sqrt{\frac{\pi z}{2}} H_{v+1 / 2}^{(i)}(z), i=1,2, \\
\psi_{v}(z)=\sqrt{\frac{\pi z}{2}} J_{v+1 / 2}(z) .
\end{gathered}
$$

$H_{v^{+}+1 / 2}^{(i)}$ is the Hankel function of $i$ th kind, order $v+\frac{1}{2}$, and argument, $z$, and $J_{v+1 / 2}$ is Bessel's function. $\zeta_{l}^{(2)}(z)$ represents an outgoing, radial elementary spherical wave and $\zeta_{v}^{(1)}(z)$ represents an ingoing, radial, elementary spherical wave, and $\psi_{n}(z)=\frac{1}{2}\left[\zeta_{n}^{(1)}(z)+\zeta_{n}^{(2)}(z)\right]$.

$$
A_{n}=\left|\begin{array}{ccccccc}
1 & \zeta_{n}^{(2)}\left(k_{1} b\right) & \zeta_{n}^{(1)}\left(k_{1} a\right) & \zeta_{n}^{(2)}\left(k_{1} r\right) & \zeta_{n}^{(1)}\left(k_{1} a\right)-\zeta_{n}^{(1)}\left(k_{1} r\right) & \zeta_{n}^{(2)}\left(k_{1} a\right) & 0 \\
\frac{k_{1}}{k_{2}} R_{n}+\frac{n}{k_{1} a} & \zeta_{n}^{(2)}\left(k_{1} b\right) & \zeta_{n-1}^{(1)}\left(k_{1} a\right) & \zeta_{n}^{(2)}\left(k_{1} r\right) & \zeta_{n-1}^{(1)}\left(k_{1} a\right)-\zeta_{n}^{(1)}\left(k_{1} r\right) & \zeta_{n-1}^{(2)}\left(k_{1} a\right) & 0 \\
0 & \zeta_{n}^{(2)}\left(k_{1} d\right) & \zeta_{n}^{(1)}\left(k_{1} b\right) & \zeta_{n}^{(2)}\left(k_{1} r\right) & \zeta_{n}^{(1)}\left(k_{1} d\right)-\zeta_{n}^{(1)}\left(k_{1} r\right) & \zeta_{n}^{(2)}\left(k_{1} d\right) & 1 \\
0 & \zeta_{n-1}^{(2)}\left(k_{1} d\right) \zeta_{n}^{(1)}\left(k_{1} b\right) & \zeta_{n}^{(2)}\left(k_{1} r\right) & \zeta_{n-1}^{(1)}\left(k_{1} d\right)-\zeta_{n}^{(1)}\left(k_{1} r\right) & \zeta_{n-1}^{(2)}\left(k_{1} d\right) & \frac{k_{1}}{k_{3}} \rho_{n}+\frac{n}{k_{1} d}
\end{array}\right|(
$$

where

$$
R_{n}=\psi_{n}^{\prime}\left(k_{2} a\right) / \psi_{n}\left(k_{2} a\right)
$$

and

$$
\rho_{n}=\zeta_{n}^{(2)^{\prime}}\left(k_{3} d\right) / \zeta_{n}^{(2)}\left(k_{3} d\right)
$$


Finally

$$
D_{n}=\left|\begin{array}{cccc}
1 & \zeta_{n}^{(2)}\left(k_{1} a\right) & \zeta_{n}^{(1)}\left(k_{1} a\right) & 0 \\
\frac{k_{1}}{k_{2}} R_{n}+\frac{n}{k_{1} a} & \zeta_{n-1}^{(2)}\left(k_{1} a\right) & \zeta_{n-1}^{(1)}\left(k_{1} a\right) & 0 \\
0 & \zeta_{n}^{(2)}\left(k_{1} d\right) & \zeta_{n}^{(1)}\left(k_{1} d\right) & 1 \\
0 & \zeta_{n-1}^{(2)}\left(k_{1} d\right) & \zeta_{n-1}^{(1)}\left(k_{1} d\right) & \frac{k_{1}}{k_{3}} \rho_{n}+\frac{n}{k_{1} d}
\end{array}\right|
$$

The zeros, $v_{s}$, of $D_{v-1 / 2}=0$, are ordered or numbered so that $\left|\operatorname{Im}\left(v_{m}\right)\right|<\left|\operatorname{Im}\left(v_{n}\right)\right|$ if $m<n$, so the residue series is convergent, since $\operatorname{Im}\left(v_{s}\right)<0$ and

$$
\frac{P_{v_{s}-1 / 2}(-\cos \theta)}{\cos v_{s} \pi} \propto\left(\frac{v_{s}}{\sin \theta}\right)^{1 / 2} \exp \left[-i\left(v_{s}\right) \theta\right],
$$

provided $\left|v_{s}\right|>>1$ and $\theta$ is not near 0 or $\pi$. Therefore, Re $\left(v_{s}\right)$ is related to the phase of a single residue, $s$, and the attenuation rate is [Attenuation] $]_{s} \cong 1.364\left|\operatorname{Im}\left(v_{s}\right)\right| \mathrm{dB} / 1000 \mathrm{~km}$.

The quantity $\frac{\left(v_{s_{1}}^{2}-1 / 4\right)\left(v_{s}\right) A_{v-1 / 2}}{\left\{\frac{\partial}{\partial v} D_{v-1 / 2}\right\}_{v=v_{s}}}$ is the excitation factor of the residue $s$. Thus this quantity weights the significance of the residue.

The expression (11) is exact except for a line integral

$$
\frac{i}{2 k_{1}^{2} r b} \int_{-i \infty}^{i \infty} \frac{v\left(v^{2}-\frac{1}{4}\right) P_{v-1 / 2}(-\cos \theta)}{\cos v \pi}\left[\frac{A_{v-1 / 2}}{D_{v-1 / 2}}-\zeta_{r-1 / 2}^{(2)}\left(k_{1} r\right) \zeta_{v-1 / 2}^{(1)}\left(k_{1} b\right)\right] d v(r \lessgtr b),
$$

which is zero if only $R_{r-1 / 2}$ is an even function of $v$ [Berry, 1964a].

This requirement $\left(R_{v-1 / 2}\right.$ even) is much less stringent than the conditions given by Wait [1963b, 1962b], i.e., that the impedance boundary conditions are exact at the ionosphere and the earth or that $\left|k_{2}\right|>>k_{1}$ and $\left|k_{3}\right|>>k_{1}$. Since (11) without the integral becomes exact as the earth's conductivity approaches infinity, the integral represents the field in the medium $a<r<d$ (the space between the ionosphere and the terrestrial sphere) resulting from waves which have escaped after traveling through the inedium $r<a$ (terrestrial sphere). The neglect of this integral (19) in terrestrial radio wave propagation is therefore a very accurate approximation, since waves traveling in the ground are very highly attenuated before again emerging into the space $a<r<d$.

\section{2. Anisotropic Solution with Constant Radial Magnetic Field}

A constant, radial magnetic field $\left(I=\mp 90^{\circ},(3)\right)$ is now imposed on the ionosphere so that it is no longer isotropic. The permittivity tensor, (3), then becomes,

$$
[\epsilon]=\epsilon_{0} i \omega\left[\begin{array}{ccc}
1-\frac{s}{s^{2}-h^{2}} & \frac{ \pm i h}{s^{2}-h^{2}} & 0 \\
\frac{\mp i h}{s^{2}-h^{2}} & 1-\frac{s}{s^{2}-h^{2}} & 0 \\
0, & 0 & 1-\frac{1}{s}
\end{array}\right]
$$

where

$$
\begin{aligned}
& s=\frac{\omega^{2}}{\omega_{N}^{2}}\left[1-i \frac{\nu}{\omega}\right], \\
& h=\frac{\omega_{H} \omega}{\omega_{N}^{2}},
\end{aligned}
$$


and where the upper or lower sign $( \pm, \mp)$ is taken for a dip angle $I=-90$ or $+90^{\circ}$, respectively (the dip or inclination angle is measured from the horizontal by convention). Wait [1963a] derived a mode equation for this model (vertical magnetic field, $I=+90^{\circ}$, i.e., directed in the $-\bar{r}$ direction). Following Wait's procedure but writing the solution first as a series of zonal harmonics, then making the Watson transformation, the Hertz-Debye potential is found in the space $a<r<d$. The vertical electric field (11) now becomes

$$
E_{r}=\frac{\pi}{k_{1}^{2} r^{2} b^{2}} \sum_{s=1}^{\infty} \frac{\left(v_{s}^{2}-\frac{1}{4}\right) v_{s} P_{v_{s}-1 / 2}(-\cos \theta)}{\cos v_{s} \pi} \frac{B_{v_{s^{-1 / 2}}}}{\left\{\frac{\partial}{\partial v} E_{v-1 / 2}\right\}_{v=v_{s}}}
$$

where $B_{v}$ and $E_{v}$ are $6 \times 6$ determinants,

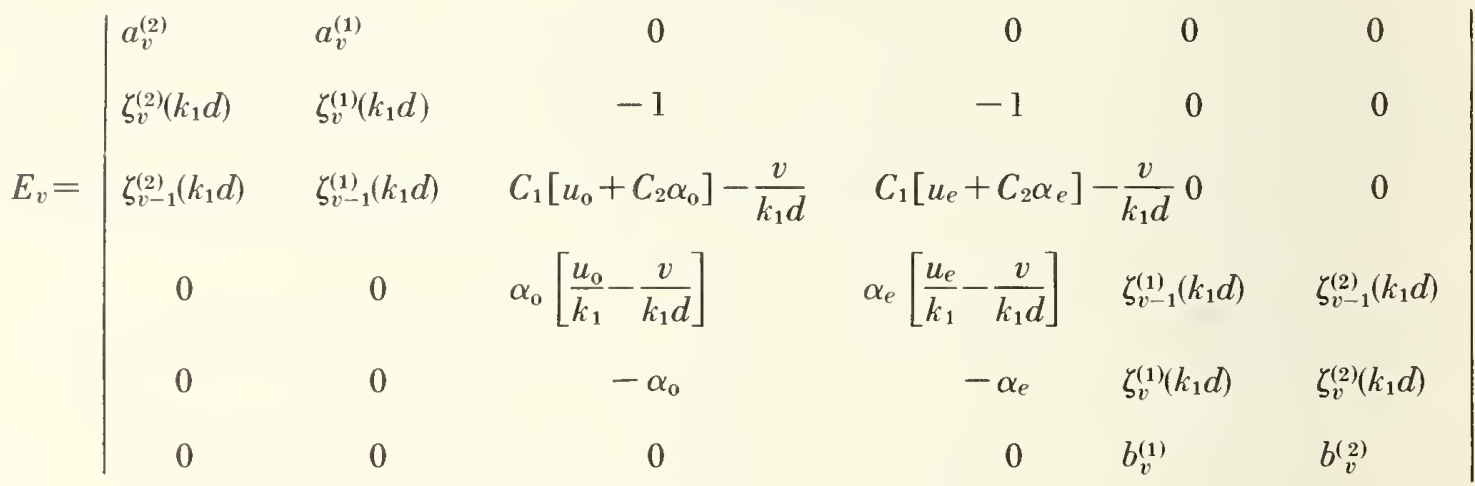

$$
\begin{aligned}
& a_{v}^{(m)}=\left[\frac{i \omega \epsilon_{0}}{k_{1}} Z_{g}+\frac{v}{k_{1} a}\right] \zeta_{v}^{(m)}\left(k_{1} a\right)-\zeta_{v-1}^{(m)}\left(k_{1} a\right), \\
& b_{v}^{(m)}=\left[\frac{i \omega \mu_{0}}{k_{1}} Y_{g}+\frac{v}{k_{1} a}\right] \zeta_{v}^{(m)}\left(k_{1} a\right)-\zeta_{v-1}^{(m)}\left(k_{1} a\right), \\
& C_{1}=\frac{\epsilon_{0} \epsilon_{1}}{k_{1} \epsilon},\left(\epsilon_{1}=\eta_{1}^{2}\right) \text { and } C_{2}=g \omega \text {. }
\end{aligned}
$$

$Z_{g}$ is the ground impedance,

$$
Z_{g}=Z_{0}\left[\frac{i \omega \epsilon_{0}}{\sigma+i \omega \epsilon_{0} \epsilon_{2}}\right]^{1 / 2}\left[1-\frac{i \omega \epsilon_{0}}{\sigma+i \omega \epsilon_{0} \epsilon_{2}}\right]^{1 / 2}
$$

and $Y_{g}$, the ground admittance,

$$
Y_{g}=\frac{1}{Z_{0}}\left[\frac{\sigma+i \omega \epsilon_{0} \epsilon_{2}}{i \omega \epsilon_{0}}\right]^{1 / 2}\left[1-\frac{i \omega \epsilon_{0}}{\sigma+i \omega \epsilon_{0} \epsilon_{2}}\right]^{1 / 2},
$$

in which $Z_{0} \sim 120 \pi \mathrm{ohms}$ is the impedance of space. $u_{0}$ and $u_{e}$ correspond to the ordinary and extraordinary waves, either magnetic or electrical solutions of an assumed planar form in the ionosphere: $A \exp \left[-u_{0}, r\right]$, where $A$ is an appropriate constant. By analogy with the Booker [1939] quartic, (6), $u_{0}$ and $u_{e}$ are solutions of

$$
u^{4}+\left(s_{1}^{2}+s_{2}^{2}\right) u^{2}+s_{1}^{2} s_{2}^{2}-s_{2}^{2} \frac{k_{1}^{2} g^{2}}{\epsilon_{0} \epsilon}=0,
$$

such that $\operatorname{Re}\left(u_{0, e}\right)>0$ (upgoing propagation components), where 


$$
\begin{aligned}
s_{1}^{2} & =k_{1}^{2} \frac{\epsilon}{\epsilon_{0}}-\lambda^{2}, \\
s_{2}^{2} & =\frac{\epsilon}{\hat{\epsilon}}\left(k^{2} \frac{\hat{\epsilon}}{\epsilon_{0}}-\lambda^{2}\right), \\
\lambda^{2} & =\frac{v^{2}-\frac{1}{4}}{d^{2}}, \\
\alpha_{0, e} & =\frac{-u_{0, e}^{2}+s_{2}^{2}}{\omega g u_{0, e}}, \\
\frac{\epsilon}{\epsilon_{0}} & =1-\frac{s}{s^{2}-h^{2}}, \\
\frac{\epsilon}{\epsilon_{0}} & =1-\frac{1}{s}, \\
\frac{g}{\epsilon_{0}} & =\frac{h}{h^{2}-s^{2}} .
\end{aligned}
$$

Equation (28) is comparable to (6), the Booker quartic, when $I=90^{\circ}, \varphi_{a}=0$.

The first two columns of $B_{v}$ are,

$$
\begin{array}{cc}
-\zeta_{v}^{(2)}\left(k_{1} b\right) a_{v}^{(1)} & \zeta_{v}^{(2)}\left(k_{1} r\right) a_{v}^{(1)}-\zeta_{v}^{(1)}\left(k_{1} r\right) a_{v}^{(2)} \\
-\zeta_{v}^{(2)}\left(k_{1} d\right) \zeta_{v}^{(1)}\left(k_{1} b\right) & \zeta_{v}^{(2)}\left(k_{1} r\right) \zeta_{v}^{(1)}\left(k_{1} d\right)-\zeta_{v}^{(1)}\left(k_{1} r\right) \zeta_{v}^{(2)}\left(k_{1} d\right) \\
-\zeta_{v-1}^{(2)}\left(k_{1} d\right) \zeta_{v}^{(1)}\left(k_{1} b\right) & \zeta_{v}^{(2)}\left(k_{1} r\right) \zeta_{v-1}^{(1)}\left(k_{1} d\right)-\zeta_{v}^{(1)}\left(k_{1} r\right) \zeta_{v-1}^{(2)}\left(k_{1} d\right) \\
0 & 0 \\
0 & 0 \\
0 & 0
\end{array}
$$

and columns 3 through 6 are the same as the corresponding columns of $E_{v},(23)$.

Equations (24) and (25) result from the use of the impedance and admittance boundary conditions [Wait, 1963a], ${ }^{3}$

$$
\begin{array}{l|l}
H_{\theta}=Y_{g} E_{\varphi} & \\
E_{\theta}=-Z_{g} H_{\varphi} & r=a .
\end{array}
$$

If the usual boundary conditions are used, $E_{v}$ and $B_{v}$ are $8 \times 8$ determinants.

$H_{r}, H_{\theta}$, and $E_{\phi}$ are no longer zero, since a weak TE field is excited by the corresponding TMfield (originally excited by the vertical electric source current) at the boundary of the anisotropic ionosphere. If the magnetic field is zero, (22) reduces to (1l) if only

$$
\rho_{l-1 / 2} \cong-i \sqrt{1-\left(\frac{v}{k_{3} d}\right)^{2}}
$$

and

$$
i \omega \epsilon_{0} Z_{g} \cong i \frac{k_{1}^{2}}{k_{2}}
$$

Equations (38) and (39) are good approximations for practical cases at VLF.

${ }^{3}$ For more details see $W$ ait [1963a]. Note that the function $\hat{h}_{r}^{(m)}(z)$ defined by Wait is identical to $\zeta^{(m)} \cdot(z)$. The latter notation is used here since it has over 50 years of historical precedence [March, 1912] and has been used by other more recent authors, e.g., Fok [1946] and Northover [1962]. 


\section{3. Reflection Coefficients}

Reflection coefficients for a plane ionosphere with plane wave incidence have been treated by Johler and Walters [1960], Johler and Harper [1962, 1963], and Johler [1963a]. A method is now discussed in which these Fresnel type reflection coefficients are introduced into the spherical wave solutions of Maxwell's equations. The $x-y$ plane becomes the surface of the ionosphere; $\hat{y}$ corresponds to $\hat{\theta}$, figure $1, \hat{z}$ to $\hat{r}$ and $\hat{x}$ to $-\hat{\phi}$. The dielectric tensor (3) is employed to deduce a reflection process for waves in the guide $(a<r<d)$.

Dimensionless field ratios are defined, Johler and Berry [1962],

$$
\begin{aligned}
& Q_{e m}^{0}=-\frac{H_{y}^{(o)}}{H_{x}^{0}} \cong \frac{H_{\theta}^{(o)}}{H_{\varphi}^{0}}, Q_{e m}^{e}=-\frac{H_{y}^{(e)}}{H_{x}^{e}} \cong \frac{H_{\theta}^{(e)}}{H_{\varphi}^{e}} \\
& Q_{m e}^{0}=-\frac{E_{x}^{(o)}}{E_{y}^{0}} \cong \frac{E_{\varphi}^{(o)}}{E_{\theta}^{0}}, Q_{m e}^{e}=-\frac{E_{x}^{(e)}}{E_{y}^{e}} \cong \frac{E_{\varphi}^{(e)}}{E_{\theta}^{e}}
\end{aligned}
$$

where $o$ and $e$ refer to ordinary and extraordinary, respectively. These ratios are functions of the angle of incidence and the connecting relationship is

$$
\sin \varphi_{i}=(n+1 / 2) / k_{1} d .
$$

The fields which satisfy Maxwell's equations (1) in the guide $(a<r<d)$, can now be written,

$$
\begin{aligned}
& E_{r}=\sum_{n} \frac{A}{k_{1}^{2}}\left[\begin{array}{l}
\zeta_{n}^{(2)}\left(k_{1} b\right) \psi_{n}\left(k_{1} r\right) \\
\psi_{n}\left(k_{1} b\right) \zeta_{n}^{(2)}\left(k_{1} r\right)
\end{array}+b_{n} \zeta_{n}^{(2)}\left(k_{1} r\right)+c_{n} \psi_{n}\left(k_{1} r\right)\right]\left(\begin{array}{l}
r<b \\
r>b
\end{array}\right) \quad(a<b<d) \\
& H_{r}=\sum_{n} \frac{A}{k_{1} \mu_{0} i \omega}\left[e_{n} \zeta_{n}^{(2)}\left(k_{1} r\right)+f_{n} \psi_{n}\left(k_{1} r\right)\right]
\end{aligned}
$$

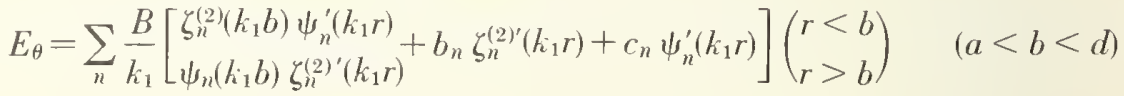

$$
\begin{aligned}
& H_{\theta}=\sum_{n} \frac{B}{\mu_{0} i \omega}\left[e_{n} \zeta_{n}^{(2)^{\prime}}\left(k_{1} r\right)+f_{n} \psi_{n}^{\prime}\left(k_{1} r\right)\right] \\
& E_{\varphi}=\sum_{n} \frac{B}{k_{1}}\left[e_{n} \zeta_{n}^{(2)}\left(k_{1} r\right)+f_{n} \psi_{n}\left(k_{1} r\right)\right] \\
& H_{\varphi}=\sum_{n} \frac{B}{\mu_{0} i \omega}\left[\begin{array}{l}
\zeta_{n}^{(2)}\left(k_{1} b\right) \psi_{n}\left(k_{1} r\right) \\
\psi_{n}\left(k_{1} b\right) \zeta_{n}^{(2)}\left(k_{1} r\right)
\end{array}+b_{n} \zeta_{n}^{(2)}\left(k_{1} r\right)+c_{n} \psi_{n}\left(k_{1} r\right)\right]\left(\begin{array}{l}
r<b \\
r>b
\end{array}\right) \quad(a<b<d)
\end{aligned}
$$

where

$$
\begin{aligned}
& A=\left[\frac{n(n+1)(2 n+1) P_{n}(\cos \theta)}{r^{2} b^{2}}\right]\left[\frac{I_{0} l \mu_{0} c}{4 \pi}\right] \\
& B=\left[\frac{(-\sin \theta)(2 n+1) P_{n}^{\prime}(\cos \theta)}{r b^{2}}\right]\left[\frac{I_{0} l \mu_{0} c}{4 \pi}\right] .
\end{aligned}
$$

For the waves in the ground, $k_{1}$ is replacer by $k_{2}(8)$, source terms containing $b_{n}$ are zero, and $\zeta_{n}^{(2)}\left(k_{2} a\right)$ waves also vanish. The constant $c_{n}$ is replaced by $a_{n}$ and $h_{n}$, the boundary constants for waves in the ground for the electrical and magnetic solution, respectively. Waves in the ionosphere are written, as the product of a field component $F$ and a boundary constant,

$$
E_{\theta, n}=d_{n} F_{\theta}^{(e)}=d_{n, 0} F_{\theta, 0}^{(e)}+d_{n, e} F_{\theta, e}^{(e)}
$$

for ordinary and extraordinary components. Similarly, 


$$
\begin{aligned}
& E_{\varphi, n}=g_{n} F_{\varphi}^{(m)} \\
& H_{\theta, n}=g_{n} F_{\theta}^{(m)} \\
& H_{\varphi, n}=d_{n} F_{\varphi .}^{(e)}
\end{aligned}
$$

In the ionosphere the known solutions of the field ratios (40) and (41) are introduced to yield boundary conditions which satisfy all tangential field types which can exist at the boundary,

$$
\begin{aligned}
& E_{\theta, 1}=E_{\theta, 3} \\
& H_{\varphi, 1}=H_{\varphi, 3} \\
& E_{\varphi, 1}=Q_{m e} E_{\theta, 3}=Q_{m e}^{(0)} E_{\theta, 3}^{(0)}+Q_{m e}^{(e)} E_{\theta, 3}^{(e)} \\
& H_{\theta, 1}=Q_{e m} H_{\varphi, 3}=Q_{e m}^{(0)} H_{\varphi, 3}^{(0)}+Q_{e m}^{(e)} H_{\varphi, 3}^{(e)} .
\end{aligned}
$$

These boundary conditions can be written in matrix form using the abbreviations $\zeta_{1 a}=\zeta_{n}^{(2)}\left(k_{1} a\right)$, $\zeta_{1 d}=\zeta_{n}^{(2)}\left(k_{1} d\right), \psi_{1 a}=\psi_{n}\left(k_{1} a\right), \psi_{1 d}=\psi_{n}\left(k_{1} d\right), \zeta_{1 b}=\zeta_{n}^{(2)}\left(k_{1} b\right), \psi_{1 b}=\psi_{n}\left(k_{1} b\right), \zeta_{2 a}=\zeta_{n}^{(2)}\left(k_{2} a\right)$, etc.

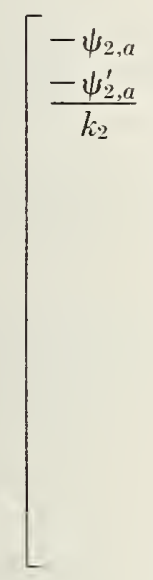

$$
\begin{array}{rrr}
\zeta_{1 a} & \psi_{1 a} & \\
\frac{\zeta_{1 a}^{\prime}}{k_{1}} & \frac{\psi_{1 a}^{\prime}}{k_{1}} & \\
\zeta_{1 d} & \psi_{1 d} & -F_{\varphi, 0}^{(e)}-F_{\varphi, e}^{(e)} \\
\frac{\zeta_{1 d}^{\prime}}{k_{1}} & \frac{\psi_{1 d}^{\prime}}{k_{1}} & -F_{\theta, 0}^{(e)}-F_{\theta, e}^{(e)} \\
& & -Q_{e m}^{(0)} F_{\varphi, 0}^{(e)}-Q_{e m}^{(e)} F_{\varphi, e}^{(e)} \\
& & -Q_{m e}^{(0)} F_{\theta, 0}^{(e)}-Q_{m e}^{(e)} F_{\varphi, e}^{(e)}
\end{array}
$$$$
\zeta_{1 d}^{\prime} \quad \psi_{1 d}^{\prime}
$$$$
\frac{\zeta_{1 d}}{k_{1}} \frac{\psi_{1 d}}{k_{1}}
$$$$
\frac{\zeta_{1 a}}{k_{1}} \frac{\psi_{1 c}}{k_{1}}-\frac{\psi_{2 a}}{k_{2}}
$$$$
\zeta_{1 a}^{\prime} \quad \psi_{1 a}^{\prime} \quad-\psi_{2 a}^{\prime}
$$$$
\left[\begin{array}{l}
a_{n} \\
b_{n} \\
c_{n} \\
d_{n, a} \\
d_{n, e} \\
e_{n} \\
f_{n} \\
h_{n}
\end{array}\right]=\left[\begin{array}{ll}
\left(-\zeta_{1 b}\right) & \psi_{1 a} \\
\left(-\zeta_{1 b}\right) & \frac{\psi_{1 d}^{\prime}}{k_{1}} \\
\left(-\psi_{1 b}\right) & \zeta_{1 d} \\
\left(-\psi_{1 b}\right) & \frac{\zeta_{1 d}^{\prime}}{k_{1}}
\end{array}\right]
$$

In the guide $(a<r<d)$ the fields which uniquely satisfy Maxwell's equations (1) for the specification of the sources, can now be determined with the aid of the boundary constants, which can be written

$$
\begin{aligned}
\psi_{1 b}+b_{n} & =\left(\psi_{1 b}-R_{e}^{s} \zeta_{1 b}\right)\left(1-R_{m}^{s} T_{m m}^{s}\right) / D_{n} \\
c_{n} & =\left(\psi_{1 b}-R_{e}^{s} \zeta_{1 b}\right)\left[-T_{e e}^{s}\left(1-R_{m}^{s} T_{m m}^{s}\right)-R_{m} T_{e m}^{s} T_{m e}^{s}\right] / D_{n} \\
e_{n} & =\left(\psi_{1 b}-R_{e}^{s} \zeta_{1 b}\right) R_{m}^{s} T_{e m}^{s} / D_{n} \\
f_{n} & =\left(\psi_{1 b}+R_{e}^{s} \zeta_{1 b}\right) T_{e m}^{s} / D_{n}
\end{aligned}
$$

where,

$$
D_{n}=\left(1-R_{e}^{s} T_{e e}^{s}\right)\left(1-R_{m}^{s} T_{m m}^{s}\right)-R_{e}^{s} R_{m}^{s} T_{e m}^{s} T_{m e}^{s}
$$

or in matrix notation, $D_{n}$ is determinant of the matrix

$$
D_{n}=\left|[1]-\left[\begin{array}{ll}
R_{e}^{s} & 0 \\
0 & R_{m}^{s}
\end{array}\right]\left[\begin{array}{ll}
T_{e e}^{s} & T_{e m}^{s} \\
T_{m e}^{s} & T_{m m}^{s}
\end{array}\right]\right|
$$


The spherical type reflection coefficients are,

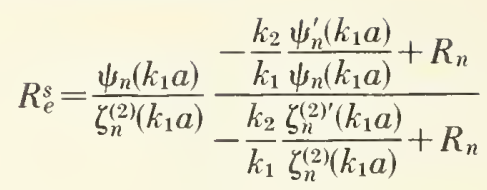

$$
\begin{aligned}
& R_{n}^{s}=\frac{\psi_{n}\left(k_{1} a\right)}{\zeta_{n}^{(2)}\left(k_{1} a\right)} \frac{\frac{-1}{k_{1}} R_{n}+\frac{1}{k_{2}} \frac{\psi_{n}^{\prime}\left(k_{1} a\right)}{\psi_{n}\left(k_{1} a\right)}}{-\frac{1}{k_{1}} R_{n}+\frac{1}{k_{2}} \frac{\zeta_{n}^{(2)^{\prime}\left(k_{1} a\right)}}{\zeta_{n}^{(2)}\left(k_{1} a\right)}} \\
& T_{e e}^{s}=\frac{\zeta_{n}^{(2)}\left(k_{1} d\right)}{\psi_{n}\left(k_{1} d\right)} T_{e e} \\
& T_{e m}^{s}=-i \frac{\zeta_{n}^{(2)}\left(k_{1} d\right)}{\psi_{n}\left(k_{1} d\right)} T_{e m} \\
& T_{m m}^{s}=\frac{\zeta_{n}^{(2)}\left(k_{1} d\right)}{\psi_{n}\left(k_{1} d\right)} T_{m m} \\
& T_{m e}^{s}=i \frac{\zeta_{n}^{(2)}\left(k_{1} d\right)}{\psi_{n}\left(k_{1} d\right)} T_{m e}
\end{aligned}
$$

where, for the case $\varphi_{a}=0$ in plane wave, plane ionosphere solution,

$$
\begin{aligned}
& T_{e e}=\Delta^{-1}\left|\begin{array}{llll}
1 & +i \eta_{0} \sqrt{\frac{Q_{m e}^{(0)}}{Q_{e m}^{(0)}}} & +i \eta_{e} \sqrt{\frac{Q_{m e}^{(e)}}{Q_{e m}^{(e)}}} & 0 \\
C_{i}\left(\zeta_{n}\right) & -1 & -1 & 0 \\
0 & -i \eta_{0} \sqrt{Q_{e m}^{(0)} Q_{m e}^{(0)}} & -i \eta_{e} \sqrt{Q_{e m}^{(e)} Q_{m e}^{(e)}} & C_{i}\left(\psi_{n}\right) \\
0 & -Q_{m e}^{(0)} & -Q_{m e}^{(e)} & 1
\end{array}\right| \\
& T_{e m}=\Delta^{-1}\left|\begin{array}{llll}
1 & +i \eta_{0} \sqrt{\frac{Q_{m e}^{(0)}}{Q_{e m}^{(0)}}} & +i \eta_{e} \sqrt{\frac{Q_{m e}^{(e)}}{Q_{e m}^{(e)}}} & 1 \\
C_{i}\left(\psi_{n}\right) & -1 & -1 & C_{i}\left(\zeta_{n}\right) \\
0 & -i \eta_{0} \sqrt{Q_{e m}^{(0)} Q_{m e}^{(0)}} & -i \eta_{e} \sqrt{Q_{e m}^{(e)} Q_{m e}^{(e)}} & 0 \\
0 & -Q_{m e}^{(0)} & -Q_{m e}^{(e)} & 0
\end{array}\right| \\
& T_{m m}=\Delta^{-1}\left|\begin{array}{llll}
1 & +i \eta_{0} \sqrt{\frac{Q_{m e}^{(0)}}{Q_{e m}^{(0)}}} & +i \eta_{e} \sqrt{\frac{Q_{m e}^{(e)}}{Q_{e m}^{(e)}}} & 0 \\
C_{i}\left(\psi_{n}\right) & -1 & -1 & 0 \\
0 & -i \eta_{0} \sqrt{Q_{e m}^{(0)} Q_{m e}^{(0)}} & -i \eta_{e} \sqrt{Q_{e m}^{(e)} Q_{m e}^{(e)}} & C_{i}\left(\zeta_{n}\right) \\
0 & -Q_{m e}^{(0)} & -Q_{m e}^{(e)} & 1
\end{array}\right| \\
& T_{m e}=\Delta^{-1}\left|\begin{array}{llll}
0 & +i \eta_{0} \sqrt{\frac{Q_{m e}^{(0)}}{Q_{e m}^{(0)}}} & +i \eta_{e} \sqrt{\frac{Q_{m e}^{(e)}}{Q_{e m}^{(e)}}} & 0 \\
0 & -1 & -1 & 0 \\
C_{i}\left(\zeta_{n}\right) & -i \eta_{0} \sqrt{Q_{e m}^{(0)} Q_{m e}^{(0)}} & -i \eta_{e} \sqrt{Q_{e m}^{(e)} Q_{m e}^{(e)}} & C_{i}\left(\psi_{n}\right) \\
1 & -Q_{m e}^{(0)} & -Q_{m e}^{(e)} & 1
\end{array}\right|
\end{aligned}
$$

and,

$$
\Delta=\left|\begin{array}{llll}
1 & +i \eta_{0} \sqrt{\frac{Q_{m e}^{(0)}}{Q_{e m}^{(0)}}} & +i \eta_{e} \sqrt{\frac{Q_{m e}^{(e)}}{Q_{e m}^{(e)}}} & 0 \\
C_{i}\left(\psi_{n}\right) & -1 & -1 & 0 \\
0 & -i \eta_{0} \sqrt{Q_{e m}^{(0)} Q_{m e}^{(0)}} & -i \eta_{e} \sqrt{Q_{e m}^{(e)} Q_{m e}^{(e)}} & C_{i}\left(\psi_{n}\right) \\
0 & -Q_{m e}^{(0)} & -Q_{m e}^{(e)} & 1
\end{array}\right|
$$




$$
\begin{aligned}
& C_{i}\left(\psi_{n}\right)=i \frac{\psi_{n}^{\prime}\left(k_{1} d\right)}{\psi_{n}\left(k_{1} d\right)} \sim \cos \phi_{i} \approx \sqrt{1-\frac{\left(v+\frac{1}{2}\right)^{2}}{\left(k_{1} d\right)^{2}}} \\
& C_{i}\left(\zeta_{n}^{(2)}\right)=i \frac{\zeta_{n}^{(2) \prime}\left(k_{1} d\right)}{\zeta_{n}^{(2)}\left(k_{1} d\right)} \sim-\cos \phi_{i} \approx-\sqrt{1-\frac{\left(v+\frac{1}{2}\right)^{2}}{\left(k_{1} d\right)^{2}}}
\end{aligned}
$$

and in the Watson transformation, $n=v_{s-1 / 2}$.

A vertical magnetic dipole or an elementary line magnetic current, $I_{m} l$, volts-meters, is equivalent to an elementary current loop $I_{0} O$, ampere-(meters) ${ }^{2}$, where $O$ is the area of the loop in square meters.

$$
I_{m} l=\mu_{0} i \omega I_{0} O
$$

The magnetic dipole source solution can be obtained from (43) to (48) by moving the source functions $\zeta_{1 b} \psi_{1 r}, \zeta_{1 b} \psi_{1 r}^{\prime}$, or $\zeta_{1 r} \psi_{1 b}, \zeta_{1 r}^{\prime} \psi_{1 b}$ from (43), (45), and (48) to (44), (46), and (47) respectively, and replacing $I_{0} l$ by $i k_{1} I_{0} O$. The fields propagated by such a vertical magnetic dipole are also equivalent to the fields propagated in the equatorial plane of a horizontal electric dipole.

The Watson transformation can be introduced to facilitate computation, which for the particular case of the vertical electric, $E_{r}$, field can be written,

$E_{r}=\left[\frac{\mu_{0} c}{4 \pi} I_{0} l\right]\left[\frac{-2 \pi}{k_{1}^{2} r^{2} b^{2}}\right] \sum_{s=1}^{\infty} \frac{v_{s}\left(v_{s}^{2}-\frac{1}{4}\right)}{\cos \left(v_{s} \pi\right)} P_{v_{s-1 / 2}}(-\cos \theta) \times \frac{b_{v_{s}-1 / 2}^{\prime} \zeta_{v_{s}-1 / 2}^{(2)}\left(k_{1} r\right)+c_{v_{s}-1 / 2}^{\prime} \psi_{v_{s}-1 / 2}\left(k_{1} r\right)}{\left\{\frac{\partial}{\partial v} D_{v_{s}-1 / 2}\right\}_{v=v_{s}}}$

where $b_{v}^{\prime}=D_{v} b_{v}, c_{v}^{\prime}=c_{v} D_{v}$, and the poles $v=v_{s}$ are the roots of the mode equation (53),

$$
D_{v_{s}-1 / 2}=0 \text {. }
$$

Computations can be made more convenient by introducing the exact relationship

$$
\psi_{n}(z)=\frac{1}{2}\left[\zeta_{n}^{(2)}(z)+\zeta_{n}^{(1)}(z)\right]
$$

into (51). The result is that $\psi_{n}(x)$ functions are replaced by $\zeta_{n}^{(1)}(x)$ functions in $D_{n}$ and in the definitions of $R^{s}$ and $T^{s}(54)$, (55), and the quantity in square brackets in (43) is (assuming $r=a=b$ )

$$
\left(1-\frac{\zeta_{1 a}^{(2)}}{\zeta_{1 a}^{(1)}} R_{e}^{s}\right) \zeta_{1 a}^{(1)} \zeta_{1 a}^{(2)}\left(\left(1-T_{m m}^{s} R_{m}^{s}\right)\left(1-\frac{\zeta_{1 a}^{(1)}}{\zeta_{1 a}^{(2)}} T_{e e}^{s}\right)-\frac{\zeta_{1 a}^{(1)}}{\zeta_{1 a}^{(2)}} T_{e m}^{s} T_{m e}^{s} R_{m}^{s}\right) D_{n}^{-1}
$$

which agrees with the result obtained by Wait [1963b] using impedance boundary conditions instead of (50). Thus, the impedance boundary conditions [Wait, 1963b] are,

$$
\begin{aligned}
& {\left[\begin{array}{l}
E_{\theta} \\
E_{\phi}
\end{array}\right]=\left[\begin{array}{rr}
Z_{11}-Z_{12} \\
-Z_{21} & Z_{22}
\end{array}\right]\left[\begin{array}{l}
H_{\theta} \\
H_{\phi}
\end{array}\right]} \\
& {\left[\begin{array}{l}
E_{y} \\
E_{x}
\end{array}\right]=\left[\begin{array}{cc}
Z_{11} & Z_{12} \\
Z_{21} & Z_{22}
\end{array}\right]\left[\begin{array}{l}
H_{y} \\
H_{x}
\end{array}\right]}
\end{aligned}
$$

where the impedance $Z_{i j}$ must be expressed with reflection coefficients and the cosine of the angle of incidence $C_{i}$,

$$
\begin{aligned}
& Z_{11}=-2 T_{m e} Z_{0} / D \\
& Z_{12}=-C_{i} Z_{0}\left[\left(1-T_{m m}\right)\left(1-T_{e e}\right)-T_{e m} T_{m e}\right] / D \\
& Z_{21}=Z_{0}\left[\left(1+T_{m m}\right)\left(1+T_{e e}\right)-T_{e m} T_{m e}\right] / D C_{i} \\
& Z_{22}=-2 T_{e m} Z_{0} / D
\end{aligned}
$$

where $D=\left(1-T_{m m}\right)\left(1+T_{e e}\right)+T_{e m} T_{m e}$. It is, therefore, concluded that the impedance boundary conditions and the conditions stated $(50)$ are equivalent.

The analysis leading to (43) to (48) and (62) is clear for propagation in the guide $(a<r<d)$ subject to some restrictions, such as $\frac{\partial}{\partial \phi}=0, \phi_{a}=0,180^{\circ}, I= \pm 90^{\circ}$. For cases $\frac{\partial}{\partial \phi} \neq 0, \phi_{a} \neq 0$, 
$180^{\circ}, I \neq \pm 90^{\circ}$, the approximation accuracy can be tested by a summation of the spherical harmonics [Wait, 1963b] in the variable $\phi$.

\section{Computational Methods}

The solutions in section 2 were programmed for a large-scale digital computer. This allows accurate computation of the functions involved, makes summation of all significant modes feasible, and provides for study of the field variation with any parameter.

The $\zeta_{v}^{(k)}(z)$ functions are computed using (12) and [Fok, 1946]

$$
H_{v}^{(k)}(z) \cong \frac{\sqrt{-i \xi} \exp \left[(-1)^{k+1} \pi i / 6\right]}{\left(z^{2}-v^{2}\right)^{1 / 4}} H_{1 / 3}^{(k)}(-i \xi), k=1,2
$$

where $\xi=v(\tanh w-w)$, and $\cosh w=v / z$. The approximation for $H_{v}^{(k)}(z)$ involving the Airy integral function, $w_{k}(z)$, which was introduced by Fok [1946], is the first term of a series in $\left(1-\frac{v^{2}}{z^{2}}\right)$ representing the right side of (65), so it is equivalent to (65) when $v \cong z$. However, (65) is uniform over a wide range of $v$ and $z$. Berry [1964b] gives detailed instructions for computing (65) and finds the magnitude of the relative error is about $10^{-2} /|z|$.

Taking the derivative with respect to order, one finds that

$$
\begin{aligned}
\frac{\partial}{\partial v} H_{b}^{(k)}(z) \cong \frac{\sqrt{-i \xi} \exp \left[(-1)^{k+1} \pi i / 6\right]}{\left(z^{2}-v^{2}\right)^{1 / 4}}\left[\left(\frac{v}{2\left(z^{2}-v^{2}\right)}-\frac{w}{6 \xi}\right) H_{1 / 3}^{(k)}(-i \xi)\right. & \\
& \left.+i w \exp \left[(-1)^{k+1} 2 \pi i / 3\right] H_{2}(k)(-i \xi)\right] .
\end{aligned}
$$

The determinates are computed numerically by reducing them to equivalent triangular determinates using the Crout forward solution and then taking the product of the diagonal elements [Hildebrand, 1946]. Full advantage is taken of the zeroes in the corners.

Let $D_{n}(v)$ be an $n \times n$ determinate whose elements are functions of $v$, and let $D_{n}^{(i)}(v)$ be the $n \times n$ determinate which is identical to $D_{n}(v)$ except that the elements of the $i$ th row (or column) are replaced by their derivatives with respect to $v$. Then

$$
\frac{\partial}{\partial v} D_{n}(v)=\sum_{i=1}^{n} D_{n}^{(i)}(v)
$$

Equation (67) is used to evaluate the denominator of the excitation factor, and in (68) below.

Given a $k$ th approximation, $v_{s}^{(k)}$, to a zero, $v_{s}$ of $D_{v-1 / 2}$, a $(k+1)$ approximation is obtained with Newton's iteration [Hildebrand, 1956],

$$
v_{s}^{(k+1)}=v_{s}^{(k)}-\frac{D_{v_{s}-1 / 2}^{(k)}}{\frac{\partial}{\partial v} D_{v_{s}^{-3 / 2}}^{(k)}}
$$

The iteration converges quadratically to $v_{s}$ if $v_{s}^{(k)}$ is sufficiently close to $v_{s}$.

Initial approximations to the $v_{s}$ are obtained several ways. Contours of $\left|D_{v-1 / 2}\right|$ can be drawn in the complex $v$-plane and the zeros approximated graphically. This is the most foolproof way of insuring that all significant zeros are found, but is slow, tedious, and expensive.

For frequencies less than about $15 \mathrm{kc} / \mathrm{s}, k_{1} a$ is an adequate first guess for $v_{1}$, and first guesses for higher-order modes for the isotropic case can be obtained from analytic expressions given by Wait [1957].

For frequencies above $15 \mathrm{kc} / \mathrm{s}$, a first guess of $k_{1} a$ frequently converges to $v_{2}$.

To find the zeros in the anisotropic case the zeros for the corresponding isotropic case are found for both vertically and horizontally polarized source dipoles (TM and TE modes). These are 
usually adequate first approximations for the anisotropic case, as shown in table 1, which gives the zeros for $f=10 \mathrm{kc} / \mathrm{s}, h=70 \mathrm{~km}, N=630$, and $\nu=10^{7}$.

TABLE 1

\begin{tabular}{c|c|c}
\hline \hline $\begin{array}{c}\text { Isotropic } \\
\text { TM modes }\end{array}$ & $\begin{array}{c}\text { Anisotropic } \\
\text { modes }\end{array}$ & $\begin{array}{c}\text { Isotropic } \\
\text { TE modes }\end{array}$ \\
\hline $1331.50-3.20 i$ & $\begin{array}{c}1331.59-3.04 i \\
1313.68-2.14 i\end{array}$ & $1313.17-1.79 i$ \\
$1260.09-22.49 i$ & $1262.34-21.4 i$ & \\
& $1222.85-8.64 i$ & $1220.62-7.07 i$ \\
$1079.42-48.77 i$ & $1077.35-49.5 i$ & \\
& $1056.52-23.9 i$ & $1048.54-18.43 i$ \\
$760.67-70.60 i$ & $739.34-83.7 i$ & \\
\hline
\end{tabular}

If the $v_{s}$ are known for some set of parameters, they can be used as first approximations for a case in which one (or more) of the parameters has been changed by a small increment. The parameter(s) can be incremented again and again until the desired set is reached. When this is done with only one parameter, the result is the variation of the field with that parameter, which is often of interest. (See, for example, figs. 2, 3, and 11.)

The Legendre function, $P_{v}(-\cos \theta)=P_{v}[\cos (\pi-\theta)]$ is computed from $\left[F_{0}\right.$, 1946]

$$
P_{v}(\cos \theta)=\frac{\Gamma(v+1)}{\Gamma(v+3 / 2) \sqrt{2 \pi \sin \theta}}\left[e^{i \phi} G_{v+1 / 2}(1-x)+e^{-i \phi} G_{v+1 / 2}(x)\right],
$$

where

$$
\phi=\theta\left(v+\frac{1}{2}\right)-\pi / 4, x=\frac{i e^{i \theta}}{2 \sin \theta},
$$

and

$$
G_{v}(x)=1+\frac{1^{2}}{2^{2} 1 !(v+1)} x+\frac{1^{2} \cdot 3^{2}}{2^{4} 2 !(v+1)(v+2)} x^{2}+\ldots+\frac{1^{2} \cdot 3^{2} \ldots(2 n-1)^{2}}{2^{2 n} n !(v+1)(v+2) \ldots(v+n)} x^{n}+\ldots .
$$

The series $G_{v}(x)$ converges if $v$ is not a negative integer and $|x|<1$, i.e., $30^{\circ}<\theta<150^{\circ}$. If $\theta$ is outside this interval, (69) is an asymptotic representation. If only the first term of $(70)$ is used, (69) reduces to (18), and the relative error is one percent or less if $|v \sin \theta|>10$. Equation (69) is adequate at VLF for all points more than $50 \mathrm{~km}$ from the source and antipode and can be used down to $15 \mathrm{~km}$ at $30 \mathrm{kc} / \mathrm{s}$.

When $\theta$ is near $\pi$ [Magnus and Oberhettinger, 1949],

$$
P_{v}(-\cos \theta)=J_{0}(\phi)+\sin ^{2}\left(\frac{\pi-\theta}{2}\right)\left[\frac{J_{1}(\phi)}{2 \phi}-J_{2}(\phi)+\frac{\phi}{6} J_{3}(\phi)\right]+O\left(\sin ^{4}\left(\frac{\pi-\theta}{2}\right)\right)
$$
where $\phi=(2 v+1) \sin \left(\frac{\pi-\theta}{2}\right)$. Equation (71) is useful for studying fields near the antipode of the
source.

\section{Discussion}

The computer program described in section 3 was used to compute the field for a variety of parameters. Three models of the lower ionosphere (sharply bounded type) were used extensively:

Model I: $h=67.5 \mathrm{~km}, N=56$ electrons $/ \mathrm{cm}^{3}, \nu=1.6\left(10^{7}\right)$;

Model II: $h=75 \mathrm{~km}, N=160$ electrons $/ \mathrm{cm}^{3}, \nu=4.5\left(10^{6}\right)$; and

Model III: $h=70 \mathrm{~km}, N=630$ electrons $/ \mathrm{cm}^{3}, \nu=10^{7}$.

Model III is a model used by Wait [1957] (corresponding to $\omega_{r}=2\left(10^{5}\right)$ in Wait paper). In his early work, Wait [1957] used the Debye approximation [Debye, 1910; March, 1912] 


$$
\frac{\zeta_{v}^{(2)^{\prime}}(z)}{\zeta_{v}^{(2)}(z)} \cong i \sqrt{1-\left(\frac{v+\frac{1}{2}}{z}\right)^{2}},
$$

for all the values of $v$ and $z$. Equation (72) is inadequate at frequencies above approximately $10 \mathrm{kc} / \mathrm{s}$ for the first or most important mode (using Wait's terminology). It is interesting to note that the inadequacy of this approximation was discussed by March [1912] for $\sin \phi_{i}=\frac{v+\frac{1}{2}}{z} \cong 1$, yet he used the approximation. Also, von Rybczyński [1913] noted the more accurate Hankel approximation (65) and used this as a basis for more accurate corrections to March's [1912] work. However, for $z$ large and $v$ small or $v$ large and $z$ small the approximation is quite excellent. The use of the approximation (72) is implied by the use of plane reflection coefficients at the ionosphere $\frac{\zeta_{n}^{(2) '}\left(k_{1} d\right)}{\zeta_{n}^{(2)}\left(k_{1} d\right)} \cong-i \cos \phi_{i}(56)$ to $(59)$. However, in this case it is a comparatively simple matter to replace $\cos \phi_{i}$ with the functions, $\zeta_{n}^{(2) \prime}\left(k_{1} d\right) / \zeta_{n}\left(k_{1} d\right)$, instead of the approximation real $\cos \phi_{i}$ or complex $\cos \phi_{i}=\sqrt{1-\left(\frac{v+\frac{1}{2}}{k_{1} d}\right)^{2}}$.

More recently, Wait [1961] and Spies and Wait [1961] used an approximation containing Airy integrals which is nearly equivalent to (65). The characteristics of the least attenuated mode were calculated. This is quite accurate [Berry, 1964a]. However, one mode is not adequate to describe the complete field which satisfies Maxwell's equations at either intermediate or great distances and higher frequencies, figures 2, 4, 5, and 6, for daytime models of the lower ionosphere (highly absorbing models such as model I). In the case of a nighttime model even more modes are needed at all distances, figure 2. Since the prime objective of the analysis of this paper is to find the field indicated by Maxwell's equations, and develop accurate techniques to calculate such a field rather than attempt to study the physical significance of modes, all modes of significance to the calculation were summed in an ordered series.

The attenuation rate of the more important modes as a function of the electron density, $N$, in the homogeneous, isotropic model of the ionosphere is shown, figure 2, for $25,10,4$, and $1 \mathrm{kc} / \mathrm{s}$. Notice that, for a fixed frequency, a given attenuation rate can be found for as many as three different values of $N$, whose magnitudes are very roughly in the proportion $1: 10: 10^{3}$. This explains why early investigators [Watson, 1918, 1919; Bremmer, 1949] deduced a high conductivity for the ionosphere, and why values of $N$ as different as those in models I and III have been considered more recently.

At VLF, an increase in electron density can cause an increase in attenuation rate for model I (daytime-noon model) while at night the attenuation rate is decreased, figure 2. The broad, nearly flat minimum in the attenuation curves, together with the insensitivity to a magnetic field previously mentioned, helps explain the observed stable characteristics of VLF propagation in the vicinity of $10 \mathrm{kc} / \mathrm{s}$ and indicates that accurate determination of ionospheric characteristics using VLF radio waves may require sophisticated techniques. The attenuation rate seems to be most sensitive to the assumed height of the ionosphere, figure 3 . This insensitivity to the detailed characteristics of the lower ionosphere suggests use of higher frequencies, such as LF (100 kc/s) which are more sensitive to ionosphere changes rather than VLF $(10 \mathrm{kc} / \mathrm{s})$, to study experimentally and theoretically the detailed characteristics of the lower ionosphere.

Figures 4, 5, and 6 illustrate the amplitude of the field and the phase correction or phase lag, $\varphi_{c}=\left|\arg E_{r}\right|-k_{1} a \theta$, of the vertical electric field as a function of distance from the source, for $30,20,6,1$, and $0.1 \mathrm{kc} / \mathrm{s}$, for three models of the ionosphere. At $30 \mathrm{kc} / \mathrm{s}$ the field shows large oscillations, even at $7000 \mathrm{~km}$. The attenuation of the second mode is nearly as small as that of the first mode at this frequency, while the excitation factor of the first mode is $1 / 5$ to $1 / 10$ that of the second mode. Thus, the two modes interfere with each other, even at great distances. The decrease in the excitation factor of the first mode $(s=1)$ with increasing frequency was noted by Wait [1962b].

At the point at which the curve for the amplitude of the field as a function of distance shows 

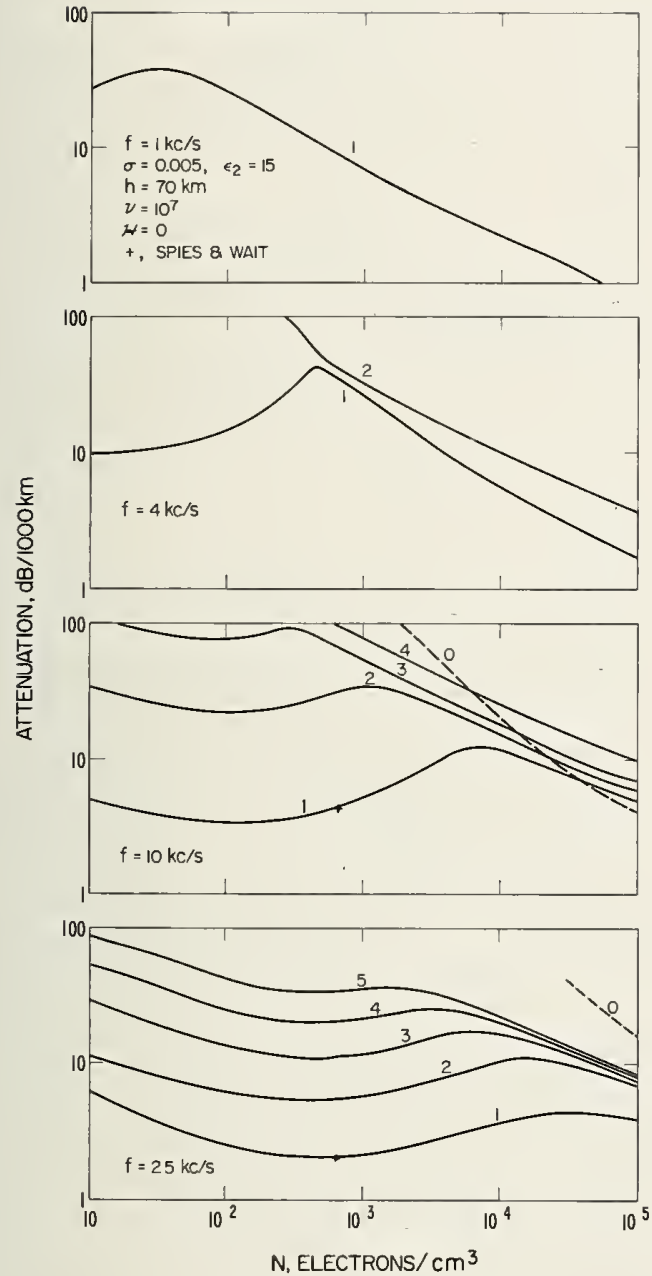

Figure 2. Attenuation rate of modes as a function of electron density, $\mathrm{N}$, at $25,10,4$, and $1 \mathrm{kc} / \mathrm{s}$.

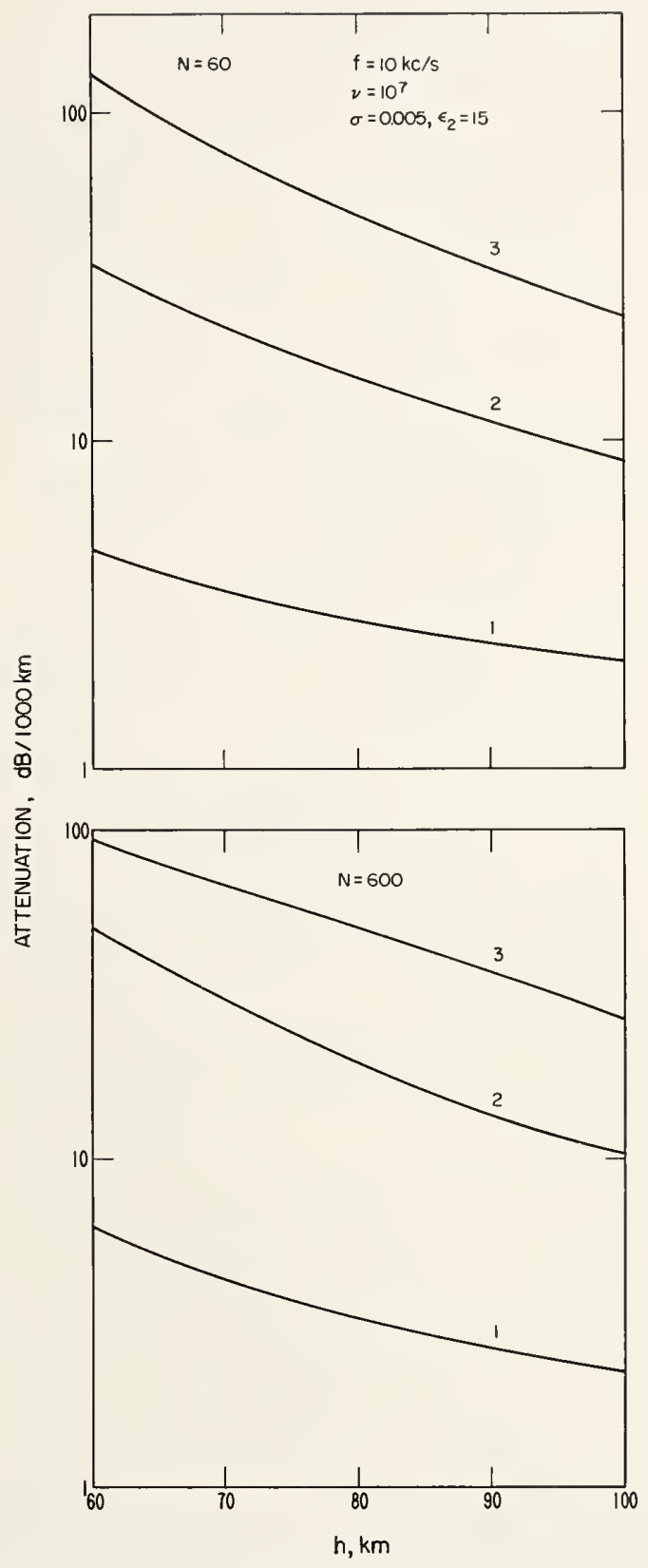

Figure 3. Attenuation rate of first three modes as a function of altitude, h, of model ionosphere boundary at $10 \mathrm{kc} / \mathrm{s}$. 

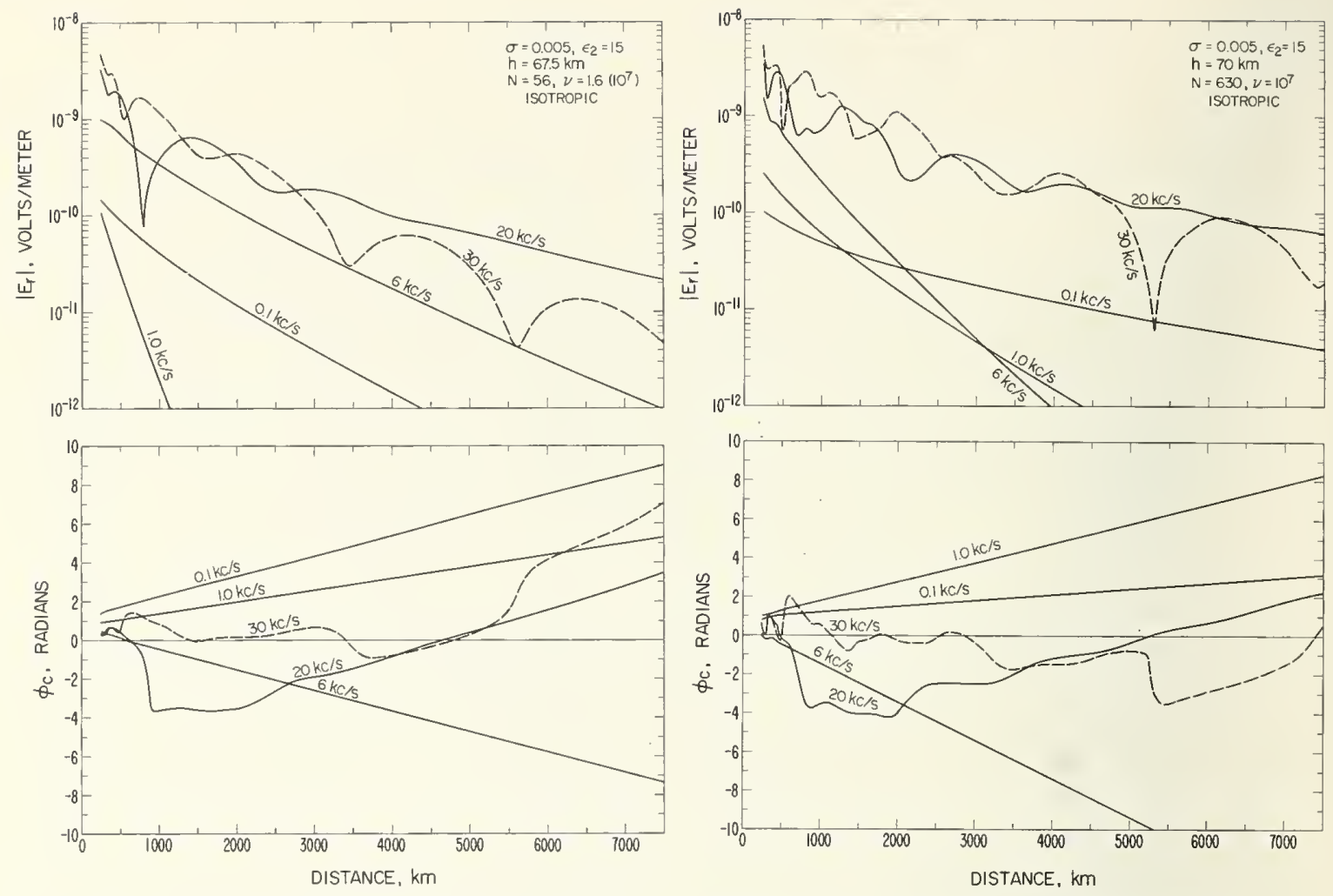

Figure 4. Amplitude and phase of the vertical electric field, $\mathrm{E}_{\mathrm{r}}$, as a function of distance for model 1 .
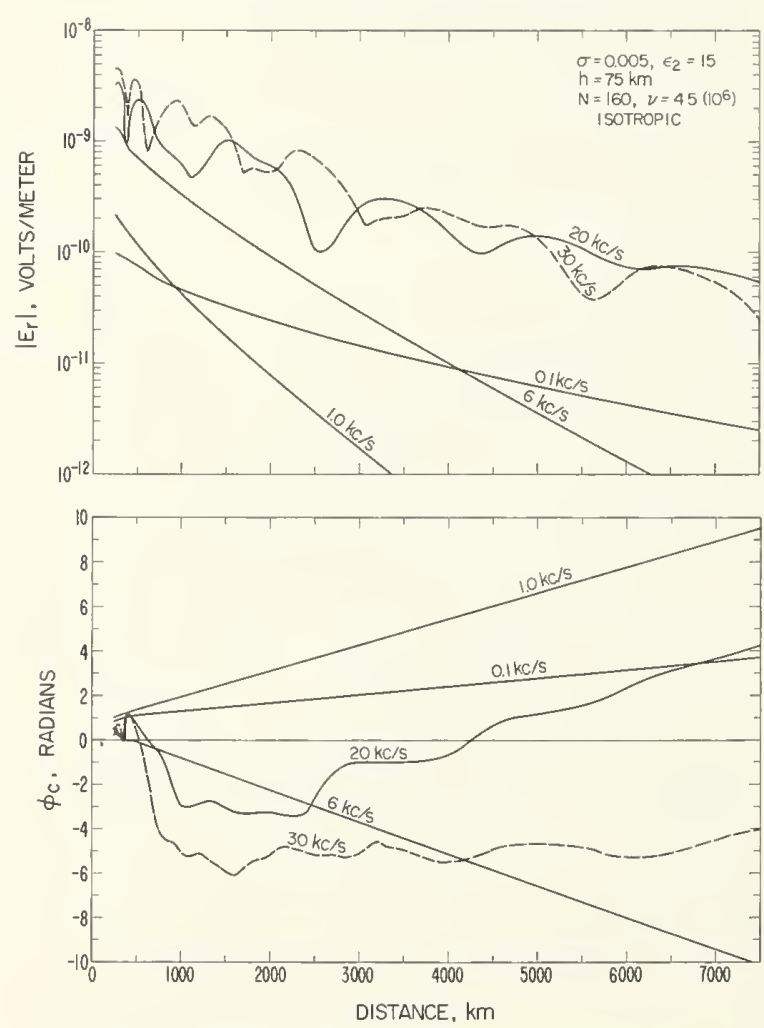

FigURE 5. Amplitude and phase of the vertical electric field, $\mathrm{E}_{\mathrm{r}}$, as a function of distance for model 11 .

Figure 6. Amplitude and phase of the vertical electric field, $\mathrm{E}_{\mathrm{r}}$, as a function of distance for model $\mathrm{III}$.
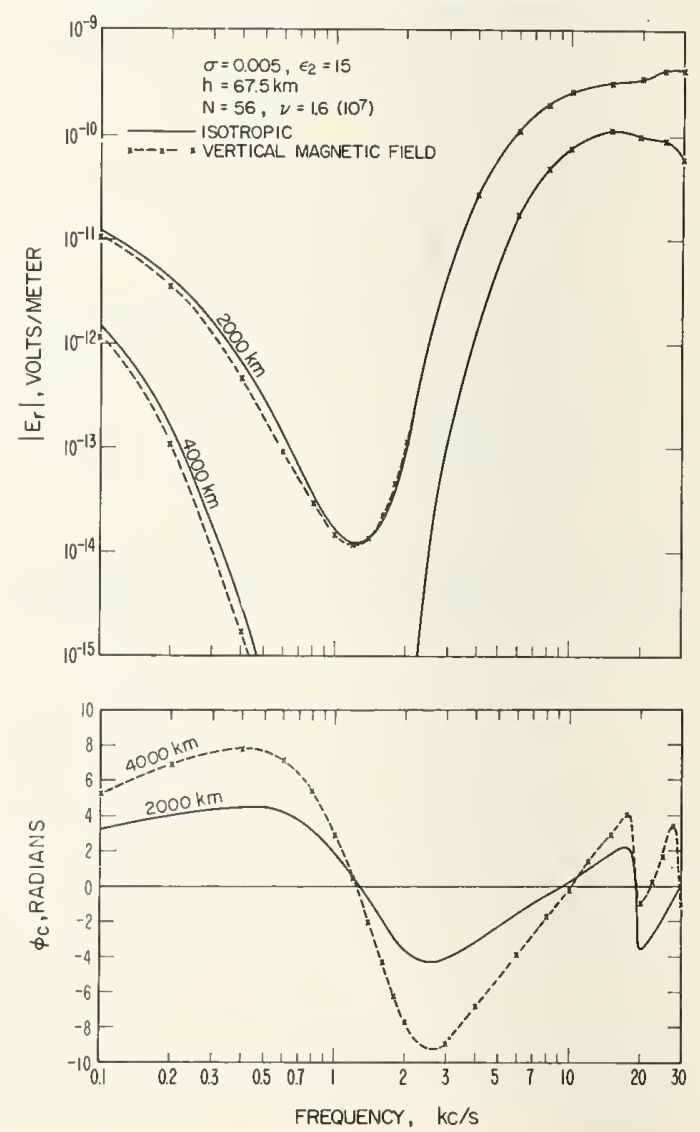

Figure 7. Amplitude and phase correction of the vertical 

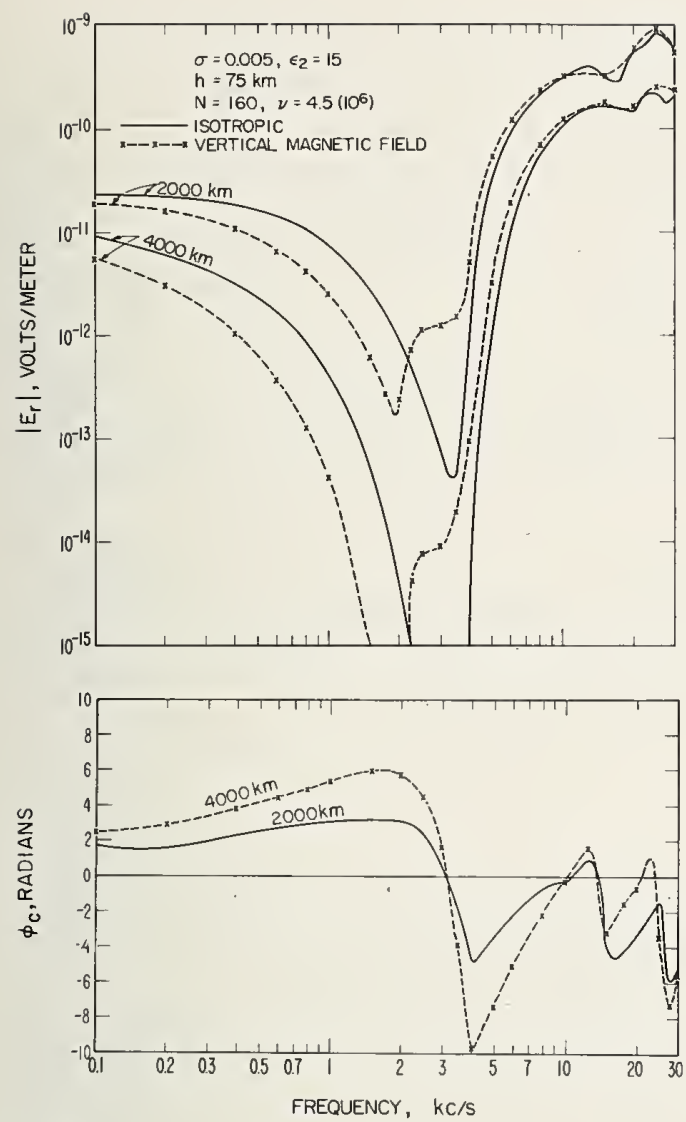

FIGURE 8. Amplitude and phase correction of the vertical electric field as a function of frequency for model II.
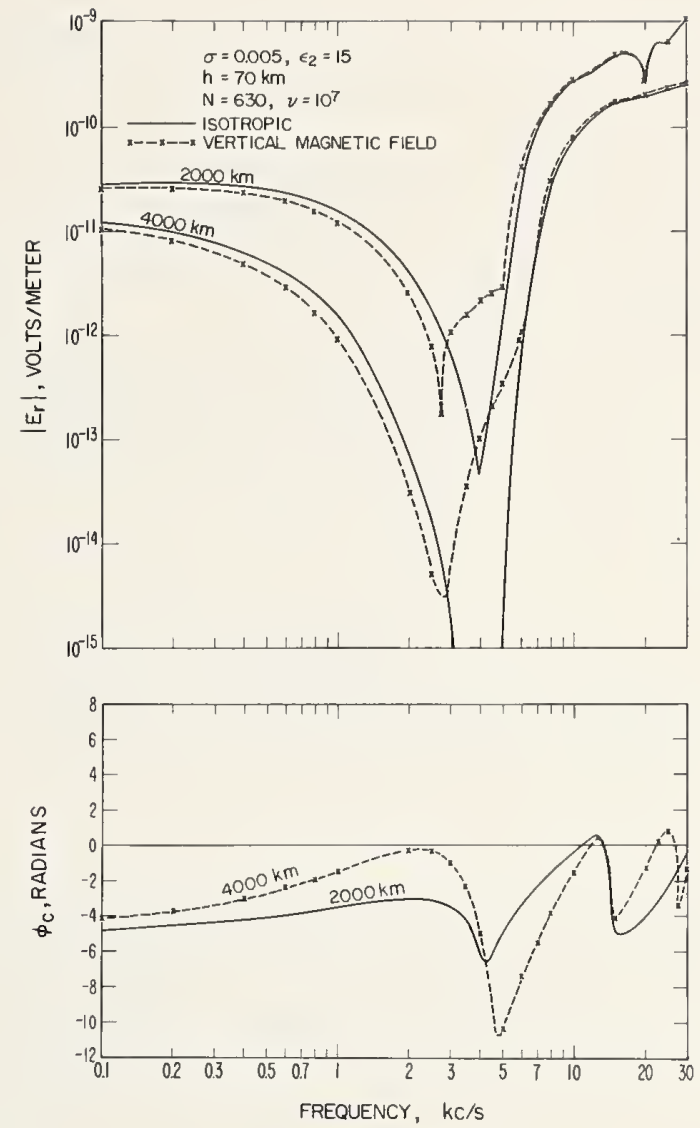

FIGURE 9. Amplitude and phase correction of the vertical electric field as a function of frequency for model III.

a sharp cusp, there is an abrupt change of approximately $\pi$ radians in the phase correction, $\phi_{c}$. This occurs in the $20 \mathrm{kc} / \mathrm{s}$ case, figure 4, at approximately $800 \mathrm{~km}$, and in the $30 \mathrm{kc} / \mathrm{s}$ case, figure 6 , at approximately $5300 \mathrm{~km}$. The cusps occur when the first two modes are exactly out of phase and have almost the same amplitude.

Figures 7, 8, and 9 show the amplitude of the field and the phase correction as a function of frequency at 2000 and $4000 \mathrm{~km}$ for the three models of the lower ionosphere both with and without a vertical magnetic field. All three models show an absorption band, the position of which can be changed by altering the assumed electron density, for example. The presence of the vertical magnetic field has a small effect in model I, figure 7, and very little effect at VLF in all models. However, figures 8 and 9 show a decrease in the field at ELF when the vertical magnetic field is present. Wait and Carter [1960] noted this effect and stated that it would occur when the collision frequency, $\nu$, is comparable with the gyrofrequency of the electron gas. Model II, figure 8, most nearly satisfies such a requirement and indeed shows the most decrease in the amplitude of the field. The presence of the static magnetic field tends to increase the amplitude of the field at VLF, figures 7, 8, and 9. Also, in figures 8 and 9, the magnetic field causes a "step" in the amplitude curve as the amplitude of the field decreases into the absorption region from higher frequencies, and the minimum field strength occurs at a lower frequency. The "step" in the curve is caused by interference between the first TE-mode (transverse electric) and the first TMmode (transverse magnetic). Ordinarily, the excitation of the first TE-mode is approximately 0.01 that of the first TM-mode. Physically, this is understandable, because the TM- or normal modes are excited by the vertical dipole sources, while the TE- or abnormal modes are coupled in at the anisotropic ionosphere boundary. However, at about 3 or $4 \mathrm{kc} / \mathrm{s}$ in models II and III, figures 8 and 9, the excitation of the first TE-mode was comparable with that of the first TM-mode, 

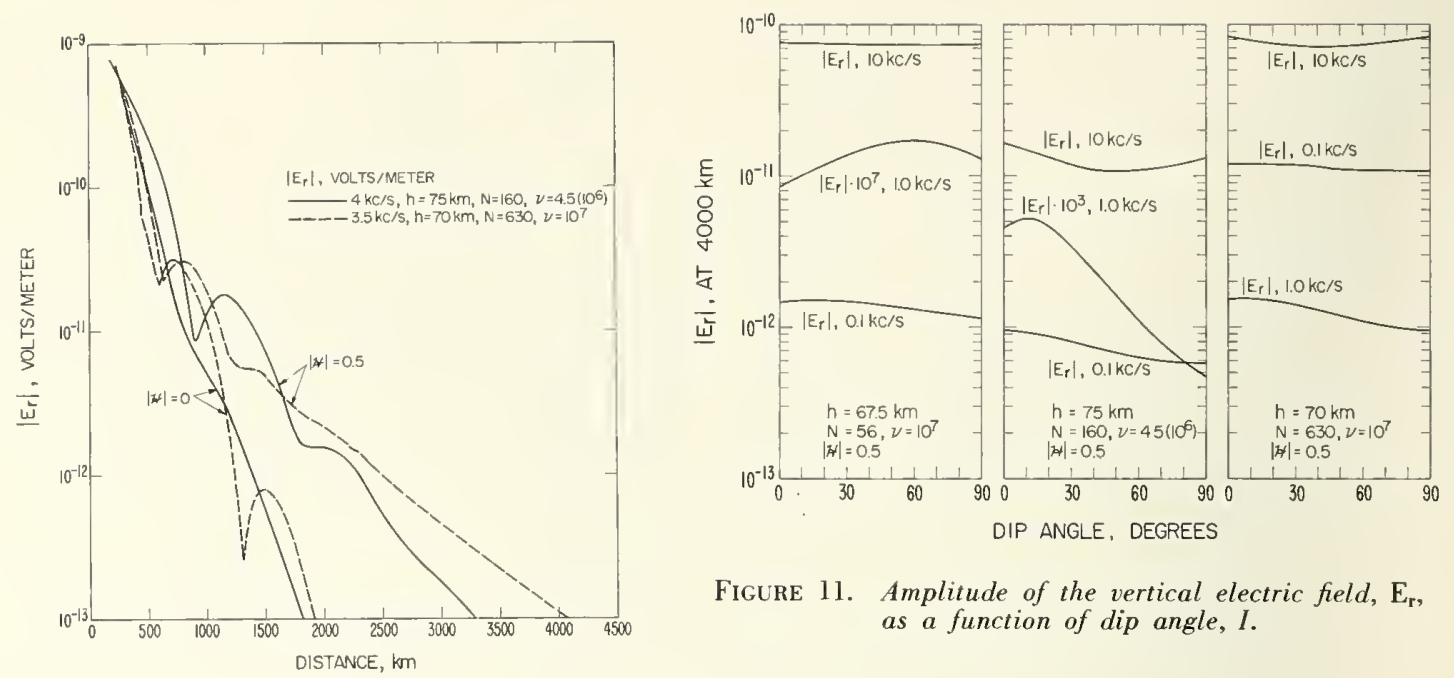

Figure 11. Amplitude of the vertical electric field, $\mathrm{E}_{\mathrm{r}}$, as a function of dip angle, $I$.

Figure 10. Amplitude of the vertical electric field, $\mathrm{E}_{\mathrm{r}}$, as a function of distance in the absorptior band.

and its attenuation rate was greater, so that they interfered with each other. Figure 10 illustrates the amplitude of the field as a function of distance for two such cases. The change in the slope of the curve clearly indicates the change in normal or abnormal mode dominance.

The effect of arbitrary dip or inclination, $I$, of the magnetic tield vector, on amplitude of the field is shown, figure 11, as a function of dip angle at 10,1 , and $0.1 \mathrm{kc} / \mathrm{s}$. The change in the amplitude of the field with dip angle is more obvious at $1 \mathrm{kc} / \mathrm{s}$. Comparison of figures 6 and 11 shows dip angles between 30 and $60 \mathrm{deg}$ exhibit virtually no difference between the isotropic and anisotropic curves at $10 \mathrm{kc} / \mathrm{s}$ (VLF).

As a consequence of the numerical and analytical insight gained by the above described analysis, the procedure to follow in introducing reflection coefficients is evident. Considerable caution is necessary since the Debye type approximation in the guide $(a<r<d)$ (64) is again not accurate for reasons described above. But in this case, there is little difficulty calculating the spherical wave functions $\zeta_{n}^{(2)}(z)$ and $\zeta_{n}^{(2)}{ }^{\prime}(z)$.

The analysis presented in this paper has been a theoretical-mathematical study based on physical premises and a number of phenomena have been predicted. Verification of these phenomena have not as yet been made under carefully controlled experiment.

\section{Conclusions}

The effect of anisotropy in the ionosphere on the field of LF, VLF, ELF radio waves can be described theoretically for the region between the ionosphere and the earth by following conventional isotropic analysis procedures and introducing the magneto-ionic theory with the aid of reflection coefficients. In addition to a considerable number of normal modes, abnormal modes must also be summed to completely describe the field. The LF portion of the long wavelength spectrum is more sensitive to ionosphere modes and changes in electron density, magnetic field, etc., than the VLF. The most important parameter at VLF is apparently the height of the model ionosphere boundary for the cases studied in this paper. The results of this paper suggests the work be extended by introducing more accurate reflection coefficients which are based on an ionosphere in which the electron density varies with altitude and distance.

The authors are indebted to their colleague, James R. Wait, for his critical review of this work. 


\section{References}

Alṕert, Ya. L. (1961), Chapter on long waves from book, Ionospheric Propagation of Radio Waves (Translation by the late Dr. Alex Nennsberg) NBS Translation T5-60, 61.

Berry, L. A. (1964a), Some remarks on the Watson transformation and mode theory, Radio Sci. J. Res. NBS/USNC-URSI $68 \mathrm{D}$, No. 1, 59-66.

Berry, L. A. (1964b), Computation of Hankel functions, NBS Tech. Note 216 (1964).

Booker, H. G. (1939), The propagation of wave packets incident obliquely on a stratified doubly refracting ionosphere, Phil. Trans. A237, 411.

Bremmer, H. (1949), Terrestrial radio waves; theory of propagation (Elsevier Publ. Co., New York, N.Y.).

Budden, K. G. (1961a), The wave-guide mode theory of wave propagation (Prentice-Hall, Inc., New Jersey).

Budden, K. G. (1961b), Radio waves in the ionosphere (Cambridge at the University Press, London).

Debye, P. (1909), Der Lichtdruck auf Kugeln von beliebigem Material, Ann. Physik (Leipzig XXX, 57-136.

Debye, P. (1910), Math. Ann. 17, p. 535 (Minutes of meeting by Academy of Science, Munich, 1910 according to March (1912).

Fok, V. A. (1946), Diffraction of radiowaves around the earth's surface, Academy of Science, USSR, Translation by Morris D. Freidman.

Hildebrand, F. B. (1946), Introduction to numerical analysis (McGraw-Hill Book Co., New York, N.Y.).

Jahnke, E., and F. Emde (1945), Tables of functions, 4th ed. (Dover Publications, New York).

Johler, J. R. (1963a), On radio wave reflections at a cont inuously stratified plasma with collisions proportional to energy and arbitrary magnetic induction, Proceedings of the International Conference on the Ionosphere, Imperial College $(2-6$ July 1962) London, England, Published by Physical Society and Institute of Physics, Chapman \& Hall Ltd, London.

Johler, J. R. (1963b), Radio pulse propagation by a reflection process at the lower ionosphere, J. Res. NBS 64D (Radio Prop.), No. 5, 481-499.

Johler, J. R., and L. A. Berry (Nov.-Dec. 1962), Propagation of terrestrial radio waves of long wavelength - theory of zonal harmonics with improved summation techniques, J. Res. NBS 66D (Radio Prop.), No. 6, 737-773.

Johler, J. R., and L. A. Berry (1963), On the propagation of long wavelength terrestrial radio waves - two theoretical techniques, Agardograph 74, Propagation of Radio Waves at Frequencies Below 300 kc/s, 101-129 (Pergamon Press, London).

Johler, J. R., and J. D. Harper, Jr. (Jan.-Feb. 1962), Reflection and transmission of radio waves at a continuously stratified plasma with arbitrary magnetic induction, J. Res. NBS 66D (Radio Prop.), No. 1, 81-99.

Johler, J. R., and J. D. Harper, Jr. (Mar. 1963), On plasma collision frequencies proportional to energy in the radio wave reflection and transmission process, NBS Tech. Note No. 164.

Johler, J. R., and L. C. Walters (May-June 1960), On the theory of reflection of low- and very-low-radiofrequency waves from the ionosphere, J. Res. NBS 64D (Radio Prop.), No. 3, 169-285.

Magnus, F., and F. Oberhettinger (1949), Special functions of mathematical physics (Chelsea Publishing Co., New York, N.Y.).

March, H. W. (1912), Über die Ausbreitung der Wellen der drahtlosen Telegraphie auf der Erdkugel, Ann. Physik, Vierte Folge XXXVII, 29-50.

Northover, F. H. (1962), The diffraction of electromagnetic waves around a finite, perfectly conduc ing cone, Quart. Journal Mech. and App. Math. XV, Pt. 1, 1-9.

Poincaré, H. (1904), Sur la diffraction des ondes electriques: à propos d' un article de M. Macdonald, Proc. Roy. Soc. London A72, 42-52.

Poincaré, H. (1910), Sur la diffraction des ondes hertziennes, Rend. Circ. Mat., Palermo, t. 29 , p. 169.

Spies, K. P., and J. R. Wait (1961), Mode calculations for VLF propagation in the earth-ionosphere waveguide, NBS Tech. Note No. 114.

von Rybczyński, W., Über die ausbreitung der Wellen der drahtlosen Telegraphie auf der Erdkugel, Ann. Physik, Vierte Folge XLI, 191-208 (Leipzig, 1913).

Wait, J. R. (June 1957), The mode theory of VLF ionospheric propagation for finite ground conductivity, Proc. IRE 45, No. 6, 760-767.

Wait, J. R. (Jan.-Feb. 1961), A new appruach to the mode theory of VLF propagation, J. Res. NBS 65D (Radio Prop.), No. 1, 37-46.

Wait, J. R. (July 1962a), Introduction to the theory of VLF propagation, Proc. IRE 50, No. 7, $1624-1648$.

Wait, J. R. (1962b), Electromagnetic waves in stratified media (Pergamon Press, Ltd., Oxford).

Wait, J. R. (May-June 1963a), Concerning solutions of the VLF mode problem for an anisotropic curved ionosphere, J. Res. NBS 67D, (Radio Prop.), No. 3, 297-302.

Wait, J. R. (1963b), The mode theory of VLF radio propagation for a spherical earth and a concentric anisotropic ionosphere, Can. J. Phys. 41, 299-315.

Wait, J. R., and N. F. Carter (Mar. 1960), Field strength calculations for ELF radio waves, NBS Tech. Note No. 52.

Wait, J. R., and A. Murphy (June 1957), The geometric optics of VLF sky wave propagation, Proc. IRE 45, No. 6, 754-760.

Wait, J. R., and K. P. Spies (Mar.-Apr. 1963), Height-gain for VLF radio waves, J. Res. NBS 67D, (Radio Prop.), No. 2, 183-187.

Watson, G. N. (1918), The diffraction of electric waves by the earth, Proc. Roy. Soc. London AXCV, 83.

Watson, G. N. (1919), The transmission of electric waves round the earth, Proc. Roy. Soc. London ACXV, 546. 


\section{Appendix}

The coefficient of the quartic (6)

$$
a_{4} \zeta^{4}+a_{3} \zeta^{3}+a_{2} \zeta^{2}+a_{1} \zeta+a_{0}=0
$$

are

$$
\begin{aligned}
& a_{0}=\left(a^{2}-1\right)^{2}\left[1-\frac{s}{s^{2}-h^{2}}\right]+\left(a^{2}-1\right)\left[\frac{1}{s}+\frac{s-2}{s^{2}-h^{2}}+\frac{a_{L}^{2} h_{T}^{2}}{s\left(s^{2}-h^{2}\right)}\right]+\frac{s-1}{s\left(s^{2}-h^{2}\right)}, \\
& a_{1}=2 \frac{h_{L} h_{T} a_{L}}{s\left(s^{2}-h^{2}\right)}\left(a^{2}-1\right), \\
& a_{2}=\left\{2\left[1-\frac{s}{s^{2}-h^{2}}\right]+\frac{h_{L}^{2}}{s\left(s^{2}-h^{2}\right)}\right\}\left(a^{2}-1\right)+\frac{h_{T}^{2} a_{L}^{2}}{s\left(s^{2}-h^{2}\right)}+\frac{s-2}{s^{2}-h^{2}}+\frac{1}{s}, \\
& a_{3}=2 \frac{h_{L} h_{T} a_{L}}{s\left(s^{2}-h^{2}\right)}=-a_{1} \sec ^{2} \phi_{i}, \\
& a_{4}=1-\frac{s^{2}-h_{L}^{2}}{s\left(s^{2}-h^{2}\right)}
\end{aligned}
$$

where,

$$
\begin{aligned}
s & =\frac{\omega^{2}}{\omega_{N}^{2}}\left[1-i \frac{\nu}{\omega}\right], \\
h & =\frac{\omega_{H} \omega}{\omega_{N}^{2}}, \\
h_{L} & =-h \sin I, \\
h_{T} & =h \cos I, \\
a_{L} & =\sin \phi_{i} \cos \phi_{a}, a_{T}=\sin \phi_{i} \sin \phi_{a}, \\
a & =\sin \phi_{i} .
\end{aligned}
$$

The solution of Maxwell's equation (1) for plane waves in a Cartesian coordinate system considered locally at the model ionosphere [Johler and Berry, 1962] and [Johler and Harper, 1962], for the dimensionless field ratios,

$$
\begin{aligned}
& P_{m e}=\frac{E_{z}}{E_{y}} \cong \frac{E_{r}}{E_{\theta}} \\
& P_{e m}=-\frac{H_{z}}{H_{x}} \cong \frac{H_{r}}{H_{\varphi}} \\
& Q_{m e}=-\frac{E_{x}}{E_{y}} \cong \frac{E_{\varphi}}{E_{\theta}} \\
& Q_{e m}=-\frac{H_{y}}{H_{x}} \cong \frac{H_{\theta}}{H_{\varphi}}
\end{aligned}
$$

can be written

$$
\left[\begin{array}{ll}
b_{11} & b_{12} \\
b_{21} & b_{22}
\end{array}\right] \quad\left[\begin{array}{l}
P_{m e} \\
Q_{m e}
\end{array}\right]+\left[\begin{array}{l}
b_{10} \\
b_{20}
\end{array}\right]=0
$$


where,

$$
\left[\begin{array}{l}
b_{11} \\
b_{12} \\
b_{21} \\
\cdot \\
b_{22} \\
b_{10} \\
b_{20}
\end{array}\right]=\left[\begin{array}{l}
a_{11} \\
a_{13} \\
a_{21} \\
a_{23} \\
a_{12} \\
a_{22}
\end{array}\right]=\left[\begin{array}{l}
a_{11} \\
a_{13} \\
a_{31} \\
a_{33} \\
a_{12} \\
a_{32}
\end{array}\right]=\left[\begin{array}{l}
a_{21} \\
a_{23} \\
a_{31} \\
\\
a_{33} \\
a_{22} \\
a_{32}
\end{array}\right],
$$

in which it has been assumed that the solution of equations (1) and (4) in Cartesian coordinates yields, upon eliminating $\vec{V}$ and $\vec{H}$,

$$
\left[\begin{array}{ccc}
a_{11} & a_{12} & a_{13} \\
a_{21} & a_{22} & a_{23} \\
a_{31} & a_{32} & a_{33}
\end{array}\right]\left[\begin{array}{l}
E_{x} \\
E_{y} \\
E_{z}
\end{array}\right]=0
$$

where,

$$
\begin{array}{rlrl}
a_{11} & =1-a_{L}^{2}-\zeta^{2}-\frac{s}{s^{2}-h^{2}}, & a_{21} & =a_{L} a_{T}+i \frac{h_{L}}{s^{2}-h^{2}} \\
a_{12} & =a_{L} a_{T}-i \frac{h_{L}}{s^{2}-h^{2}}, & a_{22} & =1-\zeta^{2}-a_{T}^{2}-\frac{s^{2}-h_{T}^{2}}{s\left(s^{2}-h^{2}\right)}, \\
a_{13} & =a_{T} \zeta+i \frac{h_{T}}{s^{2}-h^{2}}, & a_{23} & =a_{L} \zeta+\frac{h_{L} h_{T}}{s\left(s^{2}-h^{2}\right)}, \\
a_{31}=a_{T} \zeta-i \frac{h_{T}}{s^{2}-h^{2}}, & a_{32} & =a_{L} \zeta+\frac{h_{L} h_{T}}{s\left(s^{2}-h^{2}\right)}, \\
a_{33}=1-a^{2}-\frac{s^{2}-h_{L}^{2}}{s\left(s^{2}-h^{2}\right)} .
\end{array}
$$

Also,

where,

$$
\left[\begin{array}{ll}
c_{11} & c_{12} \\
c_{21} & c_{22}
\end{array}\right]\left[\begin{array}{l}
P_{e m} \\
Q_{e m}
\end{array}\right]+\left[\begin{array}{l}
c_{10} \\
c_{20}
\end{array}\right]=0
$$

$$
\left[\begin{array}{l}
c_{11} \\
c_{12} \\
c_{21} \\
c_{22} \\
c_{10} \\
c_{20}
\end{array}\right]=\left[\begin{array}{l}
d_{11} \\
d_{13} \\
d_{21} \\
d_{23} \\
d_{12} \\
d_{22}
\end{array}\right]=\left[\begin{array}{l}
d_{11} \\
d_{13} \\
d_{31} \\
d_{33} \\
d_{12} \\
d_{32}
\end{array}\right]=\left[\begin{array}{l}
d_{21} \\
d_{33} \\
d_{31} \\
d_{33} \\
d_{22} \\
d_{30}
\end{array}\right]
$$

respectively, where the şimultaneous solution of equations (1) and (4) in Cartesian coordinates yields upon eliminating $\vec{V}$ and $\vec{E}$,

$$
\left[\begin{array}{lll}
d_{11} & d_{12} & d_{13} \\
d_{21} & d_{22} & d_{23} \\
d_{31} & d_{32} & d_{33}
\end{array}\right] \cdot\left[\begin{array}{l}
H_{x} \\
H_{y} \\
H_{z}
\end{array}\right]=0
$$

where,

$$
\begin{array}{ll}
d_{11}=a_{L} A_{2} D_{c}-D_{A} \zeta\left[A_{2}^{2}-C_{3}-1\right] & d_{21}=-a_{T}\left[D_{A} \zeta^{2}+D_{B} A_{2}\right] \\
d_{12}=a_{L} D_{c}\left[\zeta^{2}-A_{1}-1\right]+a_{L} a_{T}+D_{A} \zeta & d_{22}=D_{A} B_{3} \zeta-a_{T} D_{B}\left[\zeta^{2}-A_{1}-1\right]-D_{A} D_{B} \\
d_{13}=-\left[a_{L}^{2} D_{c} \zeta+C_{1} D_{A} \zeta\right] & d_{23}=D_{A} \zeta\left[a_{T}^{2}-B_{2}-1\right]+a_{L} a_{T} D_{B} \zeta
\end{array}
$$




$$
\begin{aligned}
& d_{31}=a_{T} D_{B}\left[a_{0}^{2}-C_{3}-1\right]+a_{L} a_{T} D_{c} \zeta \\
& d_{32}=-a_{L}\left[a_{T}^{2} D_{B}-D_{c} B_{3}\right] \\
& d_{33}=a_{T} D_{B} C_{1}-a_{L} D_{c}\left[a_{T}^{2}-B_{2}-1\right]-D_{B} D_{c},
\end{aligned}
$$

where

$$
\begin{aligned}
& A_{1}=\frac{-s}{s^{2}-h^{2}} \quad A_{2}=\frac{-i h_{L}}{s^{2}-h^{2}} \quad A_{3}=\frac{i h_{T}}{s^{2}-h^{2}} \\
& B_{1}=\frac{i h_{L}}{s^{2}-h^{2}} \quad B_{2}=\frac{h_{T}^{2}-s^{2}}{s\left(s^{2}-h^{2}\right)} \quad B_{3}=\frac{h_{L} h_{T}}{s\left(s^{2}-h^{2}\right)} \\
& C_{1}=\frac{-i h_{T}}{s^{2}-h^{2}} \quad C_{2}=\frac{h_{L} h_{T}}{s\left(s^{2}-h^{2}\right)} \quad C_{3}=\frac{\left(s^{2}-h^{2}\right)}{s\left(s^{2}-h^{2}\right)}
\end{aligned}
$$

and

$$
\begin{aligned}
& D_{A}=A_{3} \zeta+a_{T} A_{1}+a_{L} A_{2}+a_{T} \\
& D_{B}=a_{T} \zeta+B_{3} \zeta+a_{L} B_{2}+a_{L} \\
& D_{C}=a_{T} C_{1}+a_{L} C_{2}+C_{3} \zeta+\zeta .
\end{aligned}
$$

In particular, explicit expressions for $Q_{m e}$ and $P_{m e}$ are,

$$
\begin{aligned}
& Q_{m e}= \frac{-\left[1-a^{2}-\frac{s^{2}-h_{L}^{2}}{s\left(s^{2}-h^{2}\right)}\right]\left[a_{L} a_{T}-i \frac{h_{L}}{s^{2}-h^{2}}\right]+\left[a_{T} \zeta+i \frac{h_{T}}{s^{2}-h^{2}}\right]\left[a_{L} \zeta+\frac{h_{L} h_{T}}{s\left(s^{2}-h^{2}\right)}\right]}{\left[1-a^{2}-\frac{s^{2}-h_{L}^{2}}{s\left(s^{2}-h^{2}\right)}\right]\left[1-a_{L}^{2}-\zeta^{2}-\frac{s}{s^{2}-h^{2}}\right]-\left[a_{T} \zeta+i \frac{h_{T}}{s^{2}-h^{2}}\right]\left[a_{T} \zeta-i \frac{h_{T}}{s^{2}-h^{2}}\right]} \\
& P_{m e}=-\left[a_{L} \zeta+\frac{h_{T} h_{L}}{s\left(s^{2}-h^{2}\right)}\right]\left[1-a_{L}^{2}-\zeta^{2}-\frac{s}{s^{2}-h^{2}}\right]+\left[a_{L} a_{T}-i \frac{h_{L}}{s^{2}-h^{2}}\right]\left[a_{T} \zeta-i \frac{h_{T}}{s^{2}-h^{2}}\right] \\
& {\left[1-a^{2}-\frac{s^{2}-h_{L}^{2}}{s\left(s^{2}-h^{2}\right)}\right]\left[1-a_{L}^{2}-\zeta^{2}-\frac{s}{s^{2}-h^{2}}\right]-\left[a_{T} \zeta+i \frac{h_{T}}{s^{2}-h^{2}}\right]\left[a_{T} \zeta-i \frac{h_{T}}{s^{2}-h^{2}}\right] }
\end{aligned}
$$


All of the equations and text of this publication were composed on the keyboard of a photocomposition system, eliminating all hand insertion of equation characters. 
